\title{
On the Singular Part of the Partition Monoid
}

\author{
James East* \\ School of Mathematics and Statistics, University of Sydney, New South Wales 2006, Australia
}

December 10, 2009

\begin{abstract}
We study the singular part of the partition monoid $\mathcal{P}_{n}$; that is, the ideal $\mathcal{P}_{n} \backslash \mathcal{S}_{n}$, where $\mathcal{S}_{n}$ is the symmetric group. Our main results are presentations in terms of generators and relations, and we also show that $\mathcal{P}_{n} \backslash \mathcal{S}_{n}$ is idempotent generated, and that its rank and idempotent-rank are both equal to $\left(\begin{array}{c}n+1 \\ 2\end{array}\right)=\frac{1}{2} n(n+1)$. One of our presentations uses an idempotent generating set of this minimal cardinality.

Keywords: Partition monoids, Transformation semigroups, Symmetric inverse semigroups, Presentations.
\end{abstract}

MSC: 20M05; 20M20.

\section{Introduction}

Let $X$ be a set and $\mathscr{T}_{X}$ the (full) transformation semigroup on $X$, which consists of all transformations on $X$ (i.e. all self-maps of $X$ ) under composition. Let $S$ be a semigroup, and let $S^{1}=S$ is $S$ has an identity, or otherwise $S^{1}=S \sqcup\{1\}$ where 1 is an adjoined identity. Cayley's Theorem (see [15] or [17] for example) states that the map $\varphi: S \rightarrow \mathscr{T}_{S^{1}}: s \mapsto \varphi_{s}$ determined by $\varphi_{s}: S^{1} \rightarrow S^{1}: t \mapsto t s$ is an embedding. If $S=S^{1}$, then $\varphi$ maps invertible elements of $S$ to invertible elements of $\mathscr{T}_{S}$, i.e. to permutations from the symmetric group $\mathscr{S}_{S}$, the group of units of $\mathscr{T}_{S}$. On the other hand, if $S \neq S^{1}$, then $s \varphi$ is not invertible for any $s \in S$ (the identity of $S^{1}$ is never in the image of $s \varphi$ ), so $\varphi$ maps $S$ into $\mathscr{T}_{S^{1}} \backslash \mathscr{S}_{S^{1}}$, the "singular part" of $\mathscr{T}_{S^{1}}$. (Of course this is not to say that any representation of a non-unital semigroup $S$ by transformations uses only singular transformations; for example, the free monogenic semigroup embeds in the symmetric group on $\mathbb{Z}$.) The singular part $\mathscr{T}_{X} \backslash \mathscr{S}_{X}$ of $\mathscr{T}_{X}$ is sometimes denoted by $\operatorname{Sing}_{X}$, and is a subsemigroup (indeed an ideal) of $\mathscr{T}_{X}$ if and only if $X$ is finite. (Even when $X$ is infinite, the set of non-surjective transformations

* jamese @ maths.usyd. edu . au 
does form a subsemigroup, and so too does the set of all non-injective transformations.) When $X=\{1, \ldots, n\}$ is finite, we write $\mathscr{T}_{n}=\mathscr{T}_{X}$ and $\mathscr{S}_{n}=\mathscr{S}_{X}$. The semigroup $\mathscr{T}_{n} \backslash \mathscr{S}_{n}$ was first studied in 1966 when Howie [16] showed that it is generated by its idempotents. Howie later showed in [18] that the rank and idempotent rank of $\mathscr{T}_{n} \backslash \mathscr{S}_{n}$ are both equal to $\left(\begin{array}{l}n \\ 2\end{array}\right)=\frac{1}{2} n(n-1)$, and also characterized all idempotent generating sets of this minimal cardinality. These results were generalized in [19] to semigroups of transformations of rank at most $r$, whose ranks and idempotent ranks were shown to be equal to $S(n, r)$, the Stirling number of the second kind. Defining relations for $\mathscr{T}_{n} \backslash \mathscr{S}_{n}$ were obtained in [8] with respect to a different generating set; this set did not consist of idempotents, and was not of minimal cardinality, but due to the asymmetry of the generating sets from [18], the author suspects that presentations in terms of minimal idempotent generating sets may not be particularly "natural". (Of course such presentations may be obtained by rewriting the defining relations from [8].) For further studies on $\mathscr{T}_{n} \backslash \mathscr{S}_{n}$, see for example $[1,2,20]$.

In sub-branches of semigroup theory, different semigroups play the role of $\mathscr{T}_{X}$. The WagnerPreston Theorem (see for example [21] or [24]) states that any inverse semigroup $S$ embeds in $\mathscr{I}_{S}$, the symmetric inverse semigroup on $S$. Again, when $S$ does not have an identity, $S$ is mapped into the singular part $\mathscr{I}_{S} \backslash \mathscr{S}_{S}$. Similarly, the FitzGerald-Leech Theorem [11] concerns embeddings of inverse semigroups in dual symmetric inverse semigroups $\mathscr{I}_{S}^{*}$, and the singular part $\mathscr{I}_{S}^{*} \backslash \mathscr{S}_{S}$ arises when $S$ has no identity. In [12] it was shown that $\mathscr{I}_{n} \backslash \mathscr{S}_{n}$ has rank $n+1$, which also coincides with its nilpotent rank if $n$ is even. Defining relations for $\mathscr{I}_{n} \backslash \mathscr{S}_{n}$ - again in terms of a larger more manageable generating set - were given in [6], where the submonoid of all order-preserving partial permutations played a central role. To the author's knowledge, the singular part of the dual symmetric inverse monoid $\mathscr{I}_{n}^{*}$ has not yet been studied. Further studies have been conducted into other singular semigroups of transformations (and similar objects); for example, the partial transformation semigroups [9], the Brauer monoids [23], and various semigroups of order-preserving transformations [13].

It is the purpose of the current article to consider the singular part $\mathcal{P}_{n} \backslash \mathcal{S}_{n}$ of the partition monoid $\mathcal{P}_{n}$. The partition monoid arises in its connection to the partition algebras which have a role of fundamental importance in the representation theory of the symmetric groups; see [14] for a detailed survey, and [7] for a study of presentations. Here we view $\mathcal{P}_{n}$ from a more semigroup theoretic point of view, motivated in part by the fact that $\mathcal{P}_{n}$ contains most of the semigroups described above as subsemigroups (see Section 3 for more details). Our main results include presentations for $\mathcal{P}_{n} \backslash \mathcal{S}_{n}$ in terms of generators and relations, and we also show that the rank and idempotent rank of $\mathcal{P}_{n} \backslash \mathcal{S}_{n}$ are both equal to $\left(\begin{array}{c}n+1 \\ 2\end{array}\right)=\frac{1}{2} n(n+1)$. This is reminiscent of Howie's result [18] concerning the rank of $\mathscr{T}_{n} \backslash \mathscr{S}_{n}$; however, in the $\mathcal{P}_{n} \backslash \mathcal{S}_{n}$ case, we are able to obtain a rather nice set of defining relations with respect to a particularly symmetric idempotent generating set. 


\section{Preliminaries}

Throughout this paper we will be mainly concerned with generation and presentation as semigroups, but we will from time to time have to deal with monoid presentations. Let $X$ be an alphabet (a set whose elements are called letters), and write $X^{*}$ for the free monoid on $X$, with the empty word being denoted by 1 . The free semigroup on $X$ is $X^{+}=X^{*} \backslash\{1\}$. If $R \subseteq X^{*} \times X^{*}$ [or $\left.R \subseteq X^{+} \times X^{+}\right]$then we denote by $R^{\sharp}$ the congruence on $X^{*}$ [or $X^{+}$] generated by $R$. We say that a monoid [or semigroup] $S$ has monoid [or semigroup] presentation $\langle X \mid R\rangle$ via a mapping $\phi: X^{*} \rightarrow S\left[\right.$ or $\phi: X^{+} \rightarrow S$ ] if (i) $\phi$ is an epimorphism, and (ii) $\operatorname{ker} \phi=R^{\sharp}$. If the mapping $\phi$ is "obvious" from context, then we will simply say $S$ has presentation $\langle X \mid R\rangle$, or just write $S \cong\langle X \mid R\rangle$. We will also call the elements of $X$ generators, and elements of $R$ relations; typically a relation $\left(w_{1}, w_{2}\right) \in R$ will be displayed as an equation, i.e. $w_{1}=w_{2}$.

There are a number of important conventions to note regarding lists and words. First, a list $x_{i}, \ldots, x_{j}$ is assumed to be empty if either

(i) $i>j$ and the subscripts are understood to be increasing; or

(ii) $i<j$ and the subscripts are understood to be decreasing.

Similarly, a word $x_{i} \cdots x_{j}$ is assumed to be empty if either (i) or (ii) above hold. (Such a word will always be a subword of a larger non-empty word if we are dealing with semigroup presentations.) This convention will also be employed in other situations, such as in the drawing of graphs. Secondly, we will adopt a kind of "Einstein convention" regarding subscripts, where an expression involving an unconstrained index is assumed to represent a list of expressions, one for each admissible value of the index (or indices). For example, if $X=\left\{x_{1}, \ldots, x_{k}\right\}$ is an alphabet, then

$$
x_{i}^{2}=x_{i} \quad \text { and } \quad x_{i} x_{j}=x_{j} x_{i}
$$

represents a list of $k+k^{2}$ relations which state that all elements of $X$ commute and are idempotents, while if $Y=\left\{y_{i j} \mid 1 \leq i<j \leq n\right\}$ then, in an expression such as

$$
y_{i j}^{2}=y_{i j},
$$

we are implicitly assuming that $i \neq j$ (among other things). Finally, all numbers we consider are integers, so a statement such as "let $1 \leq i \leq 5$ " should be read as "let $i$ be an integer for which $1 \leq i \leq 5$ ".

\section{The Partition Monoid $\mathcal{P}_{n}$}

The partition monoids have a variety of descriptions, but the geometric one is the one that will be most convenient here. Fix a positive integer $n$ for the remainder of the 
article, and write $\mathbf{n}$ for the finite set $\{1, \ldots, n\}$. Also write $\mathbf{n}^{\prime}=\left\{1^{\prime}, \ldots, n^{\prime}\right\}$ for a set in one-one correspondence with $\mathbf{n}$. Consider the equivalence relation on the set of all (simple, unlabelled, undirected) graphs on the vertex set $\mathbf{n} \cup \mathbf{n}^{\prime}$ under which two graphs are considered equivalent if and only if they have the same connected components. A partition on $\mathbf{n} \cup \mathbf{n}^{\prime}$ (or simply a partition) is defined to be an equivalence class of graphs under the above equivalence. In practice, we will think of a partition simply as a graph, identifying two graphs if they belong to the same equivalence class. When picturing partitions we will always arrange the vertices so that vertices $1, \ldots, n$ appear in a horizontal row (increasing from left to right) with vertices $1^{\prime}, \ldots, n^{\prime}$ directly below; see Figure 1 below for an example. The set of all partitions on $\mathbf{n} \cup \mathbf{n}^{\prime}$ is denoted by $\mathcal{P}_{n}$ and forms a monoid - the so-called partition monoid - under the operation we now describe. Let $\alpha, \beta \in \mathcal{P}_{n}$. To calculate

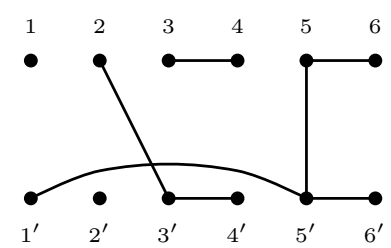

Figure 1: A partition from $\mathcal{P}_{6}$.

the product $\alpha \beta \in \mathcal{P}_{n}$, we first stack $\alpha$ and $\beta$ so that vertices $1^{\prime}, \ldots, n^{\prime}$ of $\alpha$ are identified with vertices $1, \ldots, n$ of $\beta$, then delete the middle row of vertices as well as any connected components that are not joined to an upper or lower vertex, before finally smoothing out the resulting graph on the vertex set $\mathbf{n} \cup \mathbf{n}^{\prime}$. See Figure 2 for an example calculation. It is easy enough to check that this product is associative, and that the partition $1=\lfloor\lfloor\ldots !$ with $n$ vertical edges is an identity element.

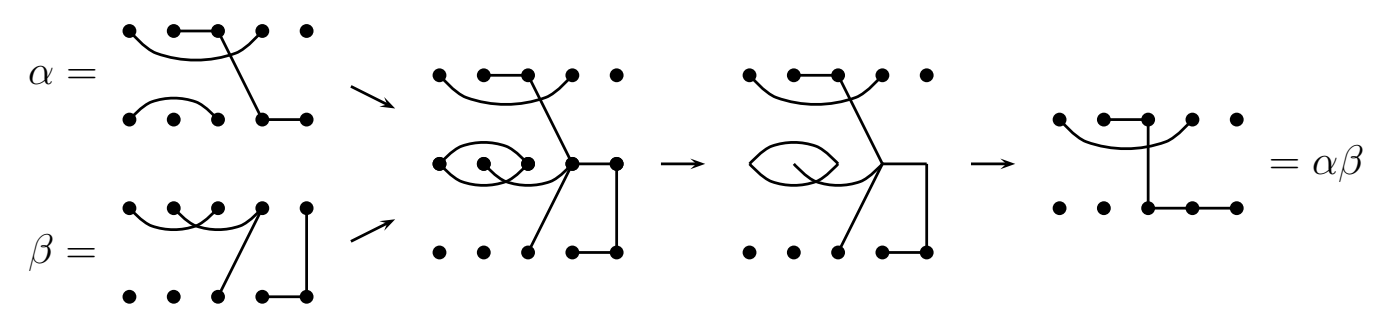

Figure 2: Calculating the product of two partitions $\alpha, \beta \in \mathcal{P}_{5}$.

There is a natural anti-involution (i.e. an anti-isomorphism of order 2)

$$
{ }^{\circ}: \mathcal{P}_{n} \rightarrow \mathcal{P}_{n}: \alpha \mapsto \alpha^{\circ}
$$

defined by reflection in a horizontal axis. More precisely, for $i, j \in \mathbf{n} \cup \mathbf{n}^{\prime},\{i, j\}$ is an edge of $\alpha$ if and only if $\left\{i^{\prime}, j^{\prime}\right\}$ is an edge of $\alpha^{\circ}$. (Here we have also written ' for the inverse bijection $\mathbf{n}^{\prime} \rightarrow \mathbf{n}$.) This map illustrates the regular structure of $\mathcal{P}_{n}$; we have $\alpha \alpha^{\circ} \alpha=\alpha$ 
and $\alpha^{\circ} \alpha \alpha^{\circ}=\alpha^{\circ}$ for all $\alpha \in \mathcal{P}_{n}$. However, $\mathcal{P}_{n}$ is not inverse if $n \geq 2$, as it is easy to find non-commuting idempotents.

We now pause to record some definitions and terminology. With this in mind, let $\alpha \in \mathcal{P}_{n}$. The connected components of (any graph representing) $\alpha$ are called its blocks and, for $i \in \mathbf{n} \cup \mathbf{n}^{\prime}$, we denote by $[i]_{\alpha} \subseteq \mathbf{n} \cup \mathbf{n}^{\prime}$ the block of $\alpha$ that contains $i$. We define the domain and codomain of $\alpha$ to be the sets

$$
\begin{aligned}
\operatorname{dom}(\alpha) & =\left\{i \in \mathbf{n} \mid[i]_{\alpha} \cap \mathbf{n}^{\prime} \neq \emptyset\right\}, \\
\operatorname{codom}(\alpha) & =\left\{i \in \mathbf{n} \mid\left[i^{\prime}\right]_{\alpha} \cap \mathbf{n} \neq \emptyset\right\} .
\end{aligned}
$$

We also define the kernel and cokernel of $\alpha$ to be the equivalences

$$
\begin{aligned}
\operatorname{ker}(\alpha) & =\left\{(i, j) \in \mathbf{n} \times \mathbf{n} \mid[i]_{\alpha}=[j]_{\alpha}\right\}, \\
\operatorname{coker}(\alpha) & =\left\{(i, j) \in \mathbf{n} \times \mathbf{n} \mid\left[i^{\prime}\right]_{\alpha}=\left[j^{\prime}\right]_{\alpha}\right\} .
\end{aligned}
$$

Basic properties include formulae such as

$$
\begin{aligned}
\operatorname{dom}\left(\alpha^{\circ}\right) & =\operatorname{codom}(\alpha), & \operatorname{ker}\left(\alpha^{\circ}\right) & =\operatorname{coker}(\alpha), \\
\operatorname{dom}(\alpha \beta) & \subseteq \operatorname{dom}(\alpha), & \operatorname{ker}(\alpha) & \subseteq \operatorname{ker}(\alpha \beta), \\
\operatorname{codom}(\alpha \beta) & \subseteq \operatorname{codom}(\beta), & \operatorname{coker}(\beta) & \subseteq \operatorname{coker}(\alpha \beta) .
\end{aligned}
$$

The equivalence classes of $\mathbf{n}$ with respect to $\operatorname{ker}(\alpha)$ and $\operatorname{coker}(\alpha)$ are called the kernelclasses and cokernel-classes of $\alpha$. A number of important semigroups of transformations (and related objects) are submonoids of the partition monoids, and may be described in terms of the above notions. Write $\Delta=\{(1,1), \ldots,(n, n)\}$ for the trivial equivalence on $\mathbf{n}$ (i.e. the equality relation). The sets

- $\mathcal{I}_{n}=\left\{\alpha \in \mathcal{P}_{n} \mid \operatorname{ker}(\alpha)=\operatorname{coker}(\alpha)=\Delta\right\}$,

- $\mathcal{I}_{n}^{*}=\left\{\alpha \in \mathcal{P}_{n} \mid \operatorname{dom}(\alpha)=\operatorname{codom}(\alpha)=\mathbf{n}\right\}$, and

- $\mathcal{T}_{n}=\left\{\alpha \in \mathcal{P}_{n} \mid \operatorname{dom}(\alpha)=\mathbf{n}\right.$ and $\left.\operatorname{coker}(\alpha)=\Delta\right\}$

are all submonoids of $\mathcal{P}_{n}$, and are isomorphic to (respectively):

- $\mathscr{I}_{n}$, the symmetric inverse semigroup on $\mathbf{n}$,

- $\mathscr{I}_{n}^{*}$, the dual symmetric inverse semigroup on $\mathbf{n}$, and

- $\mathscr{T}_{n}$, the (full) transformation semigroup on $\mathbf{n}$.

(See $[11,15,17,21,22,24]$ for further information on these semigroups.) The intersection of all three submonoids (or indeed of any two of them) is the set

- $\mathcal{S}_{n}=\left\{\alpha \in \mathcal{P}_{n} \mid \operatorname{ker}(\alpha)=\operatorname{coker}(\alpha)=\Delta\right.$ and $\left.\operatorname{dom}(\alpha)=\operatorname{codom}(\alpha)=\mathbf{n}\right\}$, 
which is isomorphic to the symmetric group $\mathscr{S}_{n}$, and is easily seen to be the group of units of $\mathcal{P}_{n}$. Without causing confusion, we will identify the semigroups $\mathscr{I}_{n}, \mathscr{T}_{n}$, etc. with their isomorphic copies inside $\mathcal{P}_{n}$. So for example, if $\alpha \in \mathcal{T}_{n}$ and $i \in \mathbf{n}$, we will sometimes write $i \alpha$ for "the image of $i$ under $\alpha$ "; that is, the unique element of $\mathbf{n}$ for which $\left\{i,(i \alpha)^{\prime}\right\}$ is an edge of $\alpha$. Note that the definition of $\mathcal{T}_{n}$ above is not symmetric, but the submonoid

- $\mathcal{T}_{n}^{\circ}=\left\{\alpha \in \mathcal{P}_{n} \mid \operatorname{codom}(\alpha)=\mathbf{n}\right.$ and $\left.\operatorname{ker}(\alpha)=\Delta\right\}$

is anti-isomorphic to $\mathcal{T}_{n}$, and will also play an important role.

\section{The singular Subsemigroup $\mathcal{P}_{n} \backslash \mathcal{S}_{n}$}

We now turn our attention to the singular part of the partition monoid $\mathcal{P}_{n}$; namely, the subsemigroup $\mathcal{P}_{n} \backslash \mathcal{S}_{n}$. But first we review a structural result regarding $\mathcal{P}_{n}$ from [7]. This will require the definition of another submonoid of $\mathcal{P}_{n}$. With this in mind, let $\alpha \in \mathcal{T}_{n}$ and suppose the kernel-classes $K_{1}, \ldots, K_{r}$ of $\alpha$ satisfy $\min \left(K_{1}\right)<\cdots<\min \left(K_{r}\right)$. We say that $\alpha$ is block-order-preserving if $K_{1} \alpha<\cdots<K_{r} \alpha$. Put

$$
\mathcal{A}_{n}=\left\{\alpha \in \mathcal{T}_{n} \mid \alpha \text { is block-order-preserving and } \operatorname{codom}(\alpha)=\mathbf{k} \text { for some } k \in \mathbf{n}\right\},
$$

which was shown to be a submonoid of $\mathcal{T}_{n}$ in [8]. Also, put $\mathcal{B}_{n}=\mathcal{A}_{n}^{\circ}$, the image of $\mathcal{A}_{n}$ under the anti-involution ${ }^{\circ}$. The following was shown in [7].

Proposition 1 Let $\alpha \in \mathcal{P}_{n}$. Then $\alpha=\beta \gamma \delta$ for unique $\beta \in \mathcal{A}_{n}, \gamma \in \mathcal{I}_{n}, \delta \in \mathcal{B}_{n}$, with $\operatorname{dom}(\gamma) \subseteq \operatorname{codom}(\beta)$ and $\operatorname{codom}(\gamma) \subseteq \operatorname{dom}(\delta)$.

The factorization of the partition from Figure 1 is given in Figure 3.

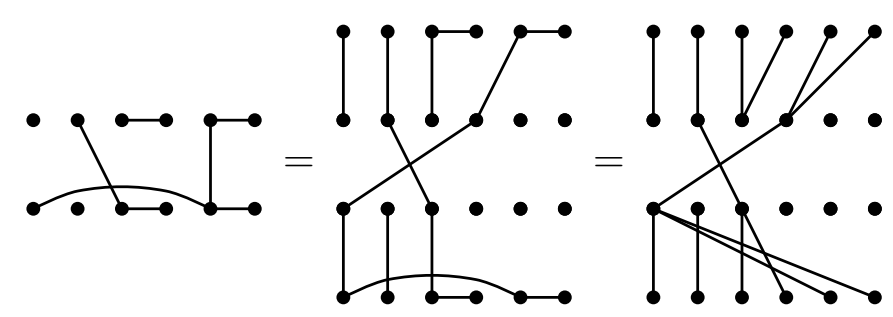

Figure 3: The factorization of a partition from $\mathcal{P}_{6}=\mathcal{A}_{6} \mathcal{I}_{6} \mathcal{B}_{6}$.

Of particular importance to us here is the following special case of Proposition 1.

Corollary 2 Let $\alpha \in \mathcal{P}_{n} \backslash \mathcal{S}_{n}$. Then $\alpha=\beta \gamma \delta$ for unique $\beta \in \mathcal{A}_{n}, \gamma \in \mathcal{I}_{n} \backslash \mathcal{S}_{n}, \delta \in \mathcal{B}_{n}$, with $\operatorname{dom}(\gamma) \subseteq \operatorname{codom}(\beta)$ and $\operatorname{codom}(\gamma) \subseteq \operatorname{dom}(\delta)$. 
Proof Let $\alpha \in \mathcal{P}_{n} \backslash \mathcal{S}_{n}$ and suppose that $\alpha=\beta \gamma \delta$ where $\beta, \gamma, \delta$ are as in Proposition 1. It suffices to show that $\gamma \in \mathcal{I}_{n} \backslash \mathcal{S}_{n}$, so suppose for the moment that $\gamma \in \mathcal{S}_{n}$. Then $\mathbf{n}=\operatorname{dom}(\gamma) \subseteq \operatorname{codom}(\beta) \subseteq \mathbf{n}$, forcing $\operatorname{codom}(\beta)=\mathbf{n}$, so that in fact $\beta \in \mathcal{S}_{n}$. Similarly, we see that $\delta \in \mathcal{S}_{n}$, so that $\alpha=\beta \gamma \delta \in \mathcal{S}_{n}$, a contradiction.

For $1 \leq i<j \leq n$, let $\bar{\alpha}_{i j} \in \mathcal{A}_{n}$ and $\bar{\beta}_{i j} \in \mathcal{B}_{n}$ denote the partitions pictured in Figure 4 . (The reason for our overline notation will become clear shortly.)
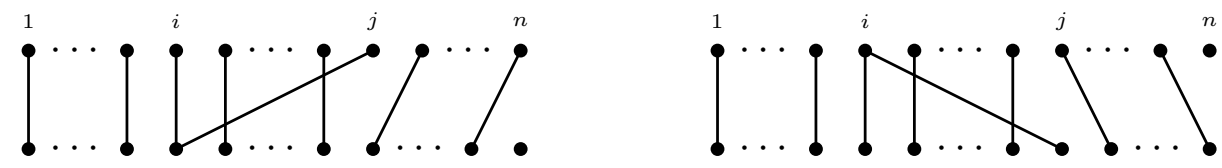

Figure 4: The partitions $\bar{\alpha}_{i j}$ (left) and $\bar{\beta}_{i j}$ (right).

It was shown in [8] that $\mathcal{A}_{n}$ is generated (as a monoid) by the set $\bar{A}=\left\{\bar{\alpha}_{i j} \mid 1 \leq i<j \leq n\right\}$, and it follows also that $\mathcal{B}_{n}$ is generated by $\bar{B}=\left\{\bar{\beta}_{i j} \mid 1 \leq i<j \leq n\right\}$. For $1 \leq i \leq n$ and $1 \leq r \leq n-2$, denote by $\bar{\lambda}_{i}, \bar{\rho}_{i}, \bar{s}_{r} \in \mathcal{I}_{n} \backslash \mathcal{S}_{n}$ the partitions pictured in Figure 5 .
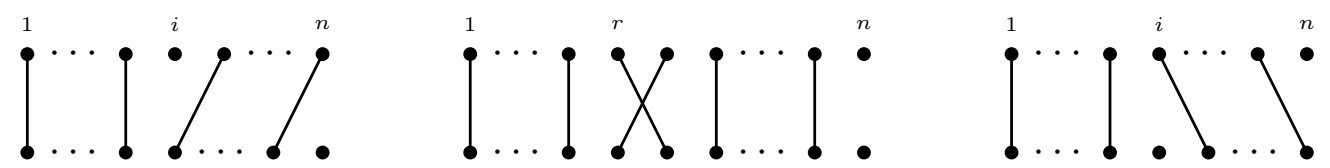

Figure 5: The partitions $\bar{\lambda}_{i}$ (left), $\bar{s}_{r}$ (middle), and $\bar{\rho}_{i}$ (right).

It was shown in [6] that $\mathcal{I}_{n} \backslash \mathcal{S}_{n}$ is generated (as a semigroup) by the set $\bar{L} \cup \bar{S} \cup \bar{R}$, where

$$
\bar{L}=\left\{\bar{\lambda}_{1}, \ldots, \bar{\lambda}_{n}\right\}, \quad \bar{S}=\left\{\bar{s}_{1}, \ldots, \bar{s}_{n-2}\right\}, \quad \bar{R}=\left\{\bar{\rho}_{1}, \ldots, \bar{\rho}_{n}\right\} .
$$

By Corollary 2, we see that $\mathcal{P}_{n} \backslash \mathcal{S}_{n}=\mathcal{A}_{n}\left(\mathcal{I}_{n} \backslash \mathcal{S}_{n}\right) \mathcal{B}_{n}$ is generated (as a semigroup) by the set $\bar{A} \cup \bar{L} \cup \bar{S} \cup \bar{R} \cup \bar{B}$. This set has $2\left(\begin{array}{l}n \\ 2\end{array}\right)+(2 n-1)+(n-2)=n^{2}+2 n-3$ elements (note that $\bar{\lambda}_{n}=\bar{\rho}_{n}$ ), and is not the smallest generating set. We will see later that the minimal size of a generating set is in fact $\left(\begin{array}{c}n+1 \\ 2\end{array}\right)=\frac{1}{2} n(n+1)$. However, this larger generating set will prove useful in deriving a system of defining relations, which can then be manipulated to yield defining relations with respect to a more compact generating set.

\section{A Presentation for $\mathcal{P}_{n} \backslash \mathcal{S}_{n}$}

In the previous section, we exhibited a factorization $\mathcal{P}_{n} \backslash \mathcal{S}_{n}=\mathcal{A}_{n}\left(\mathcal{I}_{n} \backslash \mathcal{S}_{n}\right) \mathcal{B}_{n}$ and obtained a generating set for $\mathcal{P}_{n} \backslash \mathcal{S}_{n}$ by piecing together generating sets for the three subsemigroups featuring in the factorization. We now piece together presentations for these subsemigroups 
(also necessarily adding new relations) in order to obtain defining relations for $\mathcal{P}_{n} \backslash \mathcal{S}_{n}$ in terms of the above-mentioned generating set. With this task in mind, we define alphabets

$$
\begin{gathered}
A=\left\{\alpha_{i j} \mid 1 \leq i<j \leq n\right\}, \quad B=\left\{\beta_{i j} \mid 1 \leq i<j \leq n\right\}, \\
L=\left\{\lambda_{1} \ldots, \lambda_{n}\right\}, \quad S=\left\{s_{1}, \ldots, s_{n-2}\right\}, \quad R=\left\{\rho_{1} \ldots, \rho_{n}\right\} .
\end{gathered}
$$

Consider the sets of relations

$$
\begin{aligned}
& \lambda_{i} \lambda_{j}=\lambda_{j+1} \lambda_{i} \quad \text { if } i \leq j<n \\
& \lambda_{i} \lambda_{n}=\lambda_{i} \quad \text { for all } i \\
& \rho_{j} \rho_{i}=\rho_{i} \rho_{j+1} \quad \text { if } i \leq j<n \\
& \rho_{n} \rho_{i}=\rho_{i} \quad \text { for all } i \text {; } \\
& \rho_{i} \lambda_{j}= \begin{cases}\lambda_{n} \lambda_{j-1} \rho_{i} & \text { if } i<j \\
\lambda_{n}=\rho_{n} & \text { if } i=j \\
\lambda_{n} \lambda_{j} \rho_{i-1} & \text { if } j<i\end{cases} \\
& s_{i} \lambda_{n}=s_{i} \quad \text { for all } i \\
& s_{i}^{2}=\lambda_{n} \quad \text { for all } i \\
& s_{i} s_{j}=s_{j} s_{i} \quad \text { if }|i-j|>1 \\
& s_{i} s_{j} s_{i}=s_{j} s_{i} s_{j} \quad \text { if }|i-j|=1 \text {; } \\
& s_{i} \lambda_{j}= \begin{cases}\lambda_{n} \lambda_{j} s_{i} & \text { if } i<j-1<n-1 \\
\lambda_{n} \lambda_{j-1} & \text { if } i=j-1<n-1 \\
\lambda_{n} \lambda_{j+1} & \text { if } i=j \\
\lambda_{n} \lambda_{j} s_{i-1} & \text { if } j<i\end{cases} \\
& \rho_{j} s_{i}= \begin{cases}s_{i} \rho_{j} \rho_{n} & \text { if } i<j-1<n-1 \\
\rho_{j-1} \rho_{n} & \text { if } i=j-1<n-1 \\
\rho_{j+1} \rho_{n} & \text { if } i=j \\
s_{i-1} \rho_{j} \rho_{n} & \text { if } j<i\end{cases} \\
& \alpha_{k l} \alpha_{i n}=\alpha_{k l} \quad \text { for all } i, k, l \\
& \alpha_{j k} \alpha_{i j}=\alpha_{i k} \alpha_{i j}=\alpha_{i j} \alpha_{i, k-1} \quad \text { if } i<j<k \\
& \alpha_{k l} \alpha_{i j}= \begin{cases}\alpha_{i j} \alpha_{k-1, l-1} & \text { if } i<j<k<l \\
\alpha_{i j} \alpha_{k, l-1} & \text { if } i<k<j<l \\
\alpha_{i, j+1} \alpha_{k l} & \text { if } i<k<l \leq j<n ;\end{cases}
\end{aligned}
$$




$$
\begin{array}{rlrl}
\beta_{i n} \beta_{k l} & =\beta_{k l} & & \text { for all } i, k, l \\
\beta_{i j} \beta_{j k}=\beta_{i j} \beta_{i k}= & \beta_{i, k-1} \beta_{i j} & \text { if } i<j<k \\
\beta_{i j} \beta_{k l} & = \begin{cases}\beta_{k-1, l-1} \beta_{i j} & \text { if } i<j<k<l \\
\beta_{k, l-1} \beta_{i j} & \text { if } i<k<j<l \\
\beta_{k l} \beta_{i, j+1} & \text { if } i<k<l \leq j<n .\end{cases}
\end{array}
$$

Proofs of the various parts of the following theorem may be found in [6] and [8].

Theorem 3 We have the monoid presentations

$$
\mathcal{A}_{n} \cong\langle A \mid(\mathrm{A} 1-\mathrm{A} 5)\rangle \quad \text { and } \quad \mathcal{B}_{n} \cong\langle B \mid(\mathrm{B} 1-\mathrm{B} 5)\rangle
$$

and the semigroup presentation

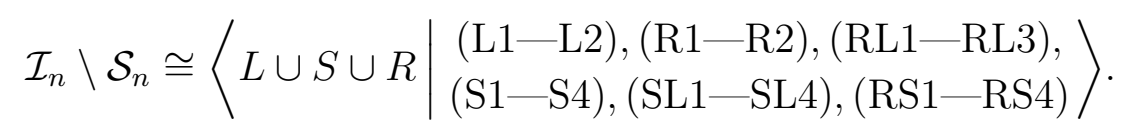

We now define an alphabet $\mathscr{X}=A \cup L \cup S \cup R \cup B$. Let $\mathscr{R}$ be the set of all the above relations, together with

$$
\begin{aligned}
\beta_{k l} \alpha_{i j}= \begin{cases}\lambda_{n} \alpha_{i-1, j-1} \beta_{k l} & \text { if } l<i \\
\lambda_{n} \alpha_{k, j-1} \beta_{k l} & \text { if } l=i \\
\lambda_{n} \alpha_{i, j-1} \beta_{k l} & \text { if } i<l<j \\
\lambda_{n} \alpha_{k i} \beta_{k i} & \text { if } k<i<j=l \\
\lambda_{n} & \text { if } k=i<j=l \\
\lambda_{n} \alpha_{i k} \beta_{i k} & \text { if } i<k<l=j \\
\lambda_{n} \alpha_{i j} \beta_{k, l-1} & \text { if } k<j<l \\
\lambda_{n} \alpha_{i j} \beta_{i, l-1} & \text { if } j=k \\
\lambda_{n} \alpha_{i j} \beta_{k-1, l-1} & \text { if } j<k ;\end{cases} \\
\lambda_{k} \alpha_{i j}= \begin{cases}\lambda_{k} & \text { if } j=n \\
\alpha_{i+1, j+1} \lambda_{k} & \text { if } k \leq i<j<n \\
\alpha_{i, j+1} \lambda_{k} & \text { if } i<k \leq j<n \\
\alpha_{i j} \lambda_{k-1} & \text { if } j<k ;\end{cases}
\end{aligned}
$$




$$
\beta_{i j} \rho_{k}=\left\{\begin{array}{l}
\rho_{k} \\
\rho_{k} \beta_{i+1, j+1} \\
\rho_{k} \beta_{i, j+1} \\
\rho_{k-1} \beta_{i j}
\end{array}\right.
$$$$
s_{r} \alpha_{i j}=\left\{\begin{array}{l}
s_{r} \\
\lambda_{n} \alpha_{i j} s_{r} \\
\lambda_{n} \alpha_{i-1, j} s_{r} \\
\lambda_{n} \alpha_{i+1, j} s_{r} \\
\lambda_{n} \alpha_{i j} \\
\lambda_{n} \alpha_{i j} s_{r} \\
\lambda_{n} \alpha_{i, j-1} \\
\lambda_{n} \alpha_{i, j+1} \\
\lambda_{n} \alpha_{i j} s_{r-1}
\end{array}\right.
$$$$
\beta_{i j} s_{r}=\left\{\begin{array}{l}
s_{r} \\
s_{r} \beta_{i j} \rho_{n} \\
s_{r} \beta_{i-1, j} \rho_{n} \\
s_{r} \beta_{i+1, j} \rho_{n} \\
\beta_{i j} \rho_{n} \\
s_{r} \beta_{i j} \rho_{n} \\
\beta_{i, j-1} \rho_{n} \\
\beta_{i, j+1} \rho_{n} \\
s_{r-1} \beta_{i j} \rho_{n}
\end{array}\right.
$$$$
\alpha_{i j} \lambda_{n}=\alpha_{i j}
$$$$
\rho_{n} \beta_{i j}=\beta_{i j}
$$

$$
\rho_{k} \alpha_{i j}= \begin{cases}\alpha_{i-1, j-1} \rho_{k} \rho_{n} & \text { if } k<i \\ s_{j-2} \cdots s_{i} & \text { if } k=i<j-1 \\ \rho_{n} & \text { if } k=i=j-1 \\ \alpha_{i, j-1} \rho_{k} \rho_{n} & \text { if } i<k<j \\ \rho_{n} & \text { if } k=j \\ \alpha_{i j} \rho_{k-1} \rho_{n} & \text { if } j<k\end{cases}
$$

if $j=n$

if $i<r=j-1$

if $r=j$

if $j<r$;

if $j=n$

if $i<r=j-1$

if $r=j$

if $j<r$;

for all $i, j$

for all $i, j$;

(BR1-BR4)

if $r<i-1<j-1<n-1$

if $r=i-1<j-1<n-1$

if $r=i<j-1<n-1$

if $r=i=j-1<n-1$

if $i<r<j-1<n-1$

if $r<i-1<j-1<n-1$

if $r=i-1<j-1<n-1$

if $r=i<j-1<n-1$

if $r=i=j-1<n-1$

if $i<r<j-1<n-1$ 


$$
\beta_{i j} \lambda_{k}= \begin{cases}\lambda_{n} \lambda_{k} \beta_{i-1, j-1} & \text { if } k<i \\ s_{i} \cdots s_{j-2} & \text { if } k=i<j-1 \\ \lambda_{n} & \text { if } k=i=j-1 \\ \lambda_{n} \lambda_{k} \beta_{i, j-1} & \text { if } i<k<j \\ \lambda_{n} & \text { if } k=j \\ \lambda_{n} \lambda_{k-1} \beta_{i j} & \text { if } j<k .\end{cases}
$$

It is our goal in this section to show that $\mathcal{P}_{n} \backslash \mathcal{S}_{n} \cong\langle\mathscr{X} \mid \mathscr{R}\rangle$. With this in mind we define

$$
\Phi: \mathscr{X}^{+} \rightarrow \mathcal{P}_{n} \backslash \mathcal{S}_{n}
$$

to be the epimorphism that extends the map $\mathscr{X} \rightarrow \mathcal{P}_{n} \backslash \mathcal{S}_{n}$ which sends each letter $\xi \in \mathscr{X}$ to its corresponding partition $\bar{\xi} \in \mathcal{P}_{n} \backslash \mathcal{S}_{n}$. We will also expand our use of the overline notation, so that for any word $w \in \mathscr{X}^{+}$we write $\bar{w}$ for the partition $w \Phi \in \mathcal{P}_{n}$. Denote by $\sim=\mathscr{R}^{\sharp}$ the congruence on $\mathscr{X}^{+}$generated by $\mathscr{R}$.

Lemma 4 We have the inclusion $\sim \subseteq \operatorname{ker} \Phi$.

Proof This follows by a straight-forward (but time-consuming) check that the relations from $\mathscr{R}$ are all preserved under $\Phi$.

The proof of the presentation $\mathcal{P}_{n} \backslash \mathcal{S}_{n} \cong\langle\mathscr{X} \mid \mathscr{R}\rangle$ makes crucial use of the following proposition which concerns factorizations of words over $\mathscr{X}$ and mirrors the structure of $\mathcal{P}_{n} \backslash \mathcal{S}_{n}=\mathcal{A}_{n}\left(\mathcal{I}_{n} \backslash \mathcal{S}_{n}\right) \mathcal{B}_{n}$ given in Corollary 2.

Proposition 5 If $w \in \mathscr{X}^{+}$, then $w \sim w_{1} w_{2} w_{3}$ for some $w_{1} \in A^{*}, w_{2} \in(L \cup S \cup R)^{+}$, $w_{3} \in B^{*}$ with $\operatorname{dom}\left(\bar{w}_{2}\right) \subseteq \operatorname{codom}\left(\bar{w}_{1}\right)$ and $\operatorname{codom}\left(\bar{w}_{2}\right) \subseteq \operatorname{dom}\left(\bar{w}_{3}\right)$.

The proof will be deferred until we have collected some intermediate technical results.

Lemma 6 Let $1 \leq i<j \leq n$ and $w \in(L \cup S \cup R \cup B)^{*}$. Then $w \alpha_{i j} \sim w_{1} w_{2}$ for some $w_{1} \in A^{*}$ and $w_{2} \in(L \cup S \cup R \cup B)^{+}$with $\ell\left(w_{1}\right) \leq 1$.

Proof First, if $\ell(w)=0$, then $w \alpha_{i j}=\alpha_{i j} \sim \alpha_{i j} \lambda_{n}$ by (AL1), so suppose that $\ell(w) \geq 1$. Write $w=w^{\prime} \xi$ where $\xi \in L \cup S \cup R \cup B$. If we can show that $\xi \alpha_{i j} \sim w_{1} w_{2}$, where $w_{1}, w_{2}$ are words of the required form, then we will be done by an induction hypothesis, seeing as $\ell\left(w^{\prime}\right)=\ell(w)-1$. Now, if $\xi \in L \cup R$, then we are done immediately, by the relevant 
relation from (LA1-LA4) or (RA1-RA6). Suppose next that $\xi=\beta_{k l} \in B$. If $k=i$ and $l=j$, then $\beta_{k l} \alpha_{i j} \sim \lambda_{n}$ by (BA5). In all other cases we have

$$
\begin{aligned}
\beta_{k l} \alpha_{i j} \sim \lambda_{n} \alpha_{p q} \beta_{r s} & \text { for some } 1 \leq p<q \leq n-1 \text { and } 1 \leq r<s \leq n, \\
& \text { by (BA1-BA4) or (BA6-BA9) } \\
\sim \alpha_{p q} \lambda_{n-1} \beta_{r s} & \text { by (LA4) }
\end{aligned}
$$

as required. Finally, the case $\xi \in S$ is covered in essentially the same way, using relations (SA1-SA9).

Corollary 7 If $w \in \mathscr{X}^{+}$, then $w \sim w_{1} w_{2}$ for some $w_{1} \in A^{*}$ and $w_{2} \in(L \cup S \cup R \cup B)^{+}$.

Proof Define $\chi: \mathscr{X}^{+} \rightarrow(\mathbb{N},+)$ to be the extension of the map

$$
\mathscr{X} \rightarrow \mathbb{N}: \xi \mapsto \begin{cases}1 & \text { if } \xi \in A \\ 0 & \text { if } \xi \in \mathscr{X} \backslash A\end{cases}
$$

The proof proceeds by induction on $k=\chi(w)$. If $k=0$ then we are already done, with $w_{1}=1$ and $w_{2}=w$, so suppose $k \geq 1$. Then $w=w_{3} \alpha_{i j} w_{4}$ for some $w_{3} \in(L \cup S \cup R \cup B)^{*}$ and $w_{4} \in \mathscr{X}^{*}$. Then

$$
\begin{aligned}
w=w_{3} \alpha_{i j} w_{4} & \sim w_{5} w_{6} w_{4} & & \text { for some } w_{5} \in A^{*} \text { and } w_{6} \in(L \cup S \cup R \cup B)^{+} \\
& \sim w_{5} w_{7} w_{2} & & \text { for some } w_{7} \in A^{*} \text { and } w_{2} \in(L \cup S \cup R \cup B)^{+},
\end{aligned}
$$

where the first equivalence follows by Lemma 6 , and the second by an induction hypothesis applied to $w_{6} w_{4}$. Since $w_{1}=w_{5} w_{7} \in A^{*}$, the proof is complete.

The proofs of the next two results are almost identical to those of Lemma 6 and Corollary 7 (respectively).

Lemma 8 Let $1 \leq i<j \leq n$ and $w \in(L \cup S \cup R)^{*}$, then $\beta_{i j} w \sim w_{1} w_{2}$ for some $w_{1} \in(L \cup S \cup R)^{+}$and $w_{2} \in B^{*}$ with $\ell\left(w_{2}\right) \leq 1$.

Corollary 9 If $w \in(L \cup S \cup R \cup B)^{+}$, then $w \sim w_{1} w_{2}$ for some $w \in(L \cup S \cup R)^{+}$and $w_{2} \in B^{*}$.

Corollary 10 If $w \in \mathscr{X}^{+}$, then $w \sim w_{1} w_{2} w_{3}$ for some $w_{1} \in A^{*}, w_{2} \in(L \cup S \cup R)^{+}$, $w_{3} \in B^{*}$. 
Proof This follows immediately from Corollaries 7 and 9.

This proves part of Proposition 5. To prove the part concerning domains and codomains, we first define words

$$
\begin{gathered}
u_{r}=\lambda_{n} \cdots \lambda_{r+1} \in L^{*}, \quad v_{r}=\rho_{r+1} \cdots \rho_{n} \in R^{*}, \\
a_{r}=\alpha_{n-1, n} \cdots \alpha_{r, r+1} \in A^{*}, \quad b_{r}=\beta_{r, r+1} \cdots \beta_{n-1, n} \in B^{*}
\end{gathered}
$$

for each $1 \leq r \leq n$, noting that all these words are empty in the case $r=n$.

Lemma 11 Let $1 \leq r \leq n$. Then $u_{r} \sim v_{r}$ and, regarding $\bar{u}_{r}$ as a partial permutation, we also have $\bar{u}_{r}=\mathrm{id}_{\mathbf{r}}$.

Proof This is essentially Lemma 26 of [6].

Lemma 12 Let $1 \leq r \leq n-1$. Then

(i) $a_{r} u_{r} \sim a_{r}$; and

(ii) $v_{r} b_{r} \sim b_{r}$.

Proof We prove (i) by (backwards) induction on $r$. The $r=n-1$ is covered by (AL1), so suppose $r<n-1$. Then

$$
\begin{aligned}
a_{r} u_{r} & =a_{r+1} \alpha_{r, r+1} \lambda_{n} \lambda_{n-1} \cdots \lambda_{r+1} & & \\
& \sim a_{r+1} \alpha_{r, r+1} \lambda_{n-1} \cdots \lambda_{r+1} & & \text { by (AL1) } \\
& \sim a_{r+1} \lambda_{n} \cdots \lambda_{r+2} \alpha_{r, r+1} & & \text { by several applications of (LA4) } \\
& =a_{r+1} u_{r+1} \alpha_{r, r+1} & & \\
& \sim a_{r+1} \alpha_{r, r+1} & & \text { by an induction hypothesis } \\
& =a_{r}, & &
\end{aligned}
$$

completing the proof of (i). Part (ii) is proved analogously.

Corollary 13 Let $1 \leq r \leq n-1$.

(i) If $w \in A^{*}$ and $\operatorname{codom}(\bar{w})=\mathbf{r}$, then $w \sim w u_{r}$.

(ii) If $w \in B^{*}$ and $\operatorname{dom}(\bar{w})=\mathbf{r}$, then $w \sim u_{r} w$. 

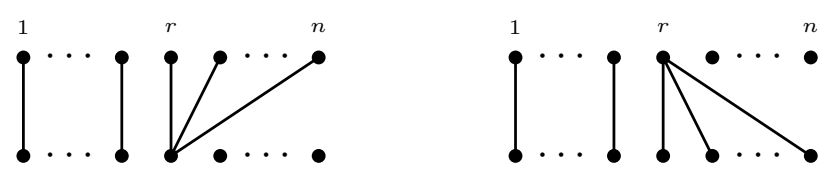

Figure 6: The partitions $\bar{a}_{r}$ (left) and $\bar{b}_{r}$ (right).

Proof Diagrammatically, it is easy to see that $\bar{w} \bar{a}_{r}=\bar{w}$; see Figure 6 for an illustration of $\bar{a}_{r}$ (and $\bar{b}_{r}$ ). It follows by Theorem 3 that $w a_{r} \sim w$. But then using this, and Lemma 12, we have

$$
w \sim w a_{r} \sim w a_{r} u_{r} \sim w u_{r}
$$

showing that (i) holds. Again, (ii) is proved in a similar fashion.

Proof of Proposition 5 Let $w \in \mathscr{X}^{+}$. By Corollary 10 we have $w \sim w_{1} w_{2}^{\prime} w_{3}$ for some $w_{1} \in A^{*}, w_{2}^{\prime} \in(L \cup S \cup R)^{+}, w_{3} \in B^{*}$. Now, $\operatorname{codom}\left(\bar{w}_{1}\right)=\mathbf{r}$ and $\operatorname{dom}\left(\bar{w}_{3}\right)=\mathbf{s}$ for some $1 \leq r, s \leq n$. Put $w_{2}=u_{r} w_{2}^{\prime} u_{s} \in(L \cup S \cup R)^{+}$. Then

$$
w \sim w_{1} w_{2}^{\prime} w_{3} \sim w_{1} u_{r} w_{2}^{\prime} u_{s} w_{3}=w_{1} w_{2} w_{3},
$$

where the second equivalence follows from (both parts of) Corollary 13 if $r$ and/or $s$ are not equal to $n$ (recall that $u_{n}=1$ ). Further, we have

$$
\operatorname{dom}\left(\bar{w}_{2}\right)=\operatorname{dom}\left(\bar{u}_{r} \bar{w}_{2}^{\prime} \bar{u}_{s}\right) \subseteq \operatorname{dom}\left(\bar{u}_{r}\right)=\mathbf{r}=\operatorname{codom}\left(\bar{w}_{1}\right),
$$

and similarly $\operatorname{codom}\left(\bar{w}_{2}\right) \subseteq \operatorname{dom}\left(\bar{w}_{3}\right)$, completing the proof.

We are now able to prove the main result of this section.

Theorem 14 We have the presentation $\mathcal{P}_{n} \backslash \mathcal{S}_{n} \cong\langle\mathscr{X} \mid \mathscr{R}\rangle$.

Proof All that remains is to show that $\operatorname{ker} \Phi \subseteq \sim$, so suppose $\left(w, w^{\prime}\right) \in \operatorname{ker} \Phi$. Now, by Proposition 5,

$$
w \sim w_{1} w_{2} w_{3} \quad \text { and } \quad w^{\prime} \sim w_{1}^{\prime} w_{2}^{\prime} w_{3}^{\prime}
$$

for appropriate $w_{i}, w_{i}^{\prime}(i=1,2,3)$. We then have

$$
\bar{w}_{1} \bar{w}_{2} \bar{w}_{3}=w \Phi=w^{\prime} \Phi=\bar{w}_{1}^{\prime} \bar{w}_{2}^{\prime} \bar{w}_{3}^{\prime} .
$$

But then by Corollary 2, we have $\bar{w}_{i}=\bar{w}_{i}^{\prime}$ for each $i$, so that each $w_{i} \sim w_{i}^{\prime}$ by the relevant presentation from Theorem 3. It follows that $w \sim w^{\prime}$, and the proof is complete. 
Remark 15 Note that the set $\overline{\mathscr{X}}$ contains some redundant generators. Indeed, $\bar{\lambda}_{n}$ and $\bar{\rho}_{n}$ as well as all of the $\bar{s}_{r}$ are unneeded since

$$
\begin{aligned}
\bar{\rho}_{n}=\bar{\lambda}_{n} & =\bar{\beta}_{12} \bar{\alpha}_{12} & & \text { by (RL2) and (BA5), and } \\
\bar{s}_{r}=\bar{\rho}_{r} \bar{\alpha}_{r, r+2} & =\bar{\beta}_{r, r+2} \bar{\lambda}_{r} & & \text { for all } r, \text { by (RA2) and (BL2). }
\end{aligned}
$$

In fact, we will see in the next section that no more generators may be removed from this set, but that the minimal size of a generating set for $\mathcal{P}_{n} \backslash \mathcal{S}_{n}$ is $\left(\begin{array}{c}n+1 \\ 2\end{array}\right)=\frac{1}{2} n(n+1)$, as compared with the $2\left(\begin{array}{l}n \\ 2\end{array}\right)+2(n-1)=n^{2}+n-2$ elements of this irreducible generating set.

\section{The Rank of $\mathcal{P}_{n} \backslash \mathcal{S}_{n}$}

Recall that the rank of a semigroup $S$, denoted $\operatorname{rank}(S)$, is the minimal cardinality of a subset of $S$ which generates it (as a semigroup). If $S$ is generated by its idempotents, then the idempotent rank, denoted id-rank $(S)$, is the minimal cardinality of a set of idempotents that generates $S$ (as a semigroup). It is our aim in this section to show that $\mathcal{P}_{n} \backslash \mathcal{S}_{n}$ is idempotent generated, and that $\operatorname{rank}(S)=\mathrm{id}-\operatorname{rank}(S)=\left(\begin{array}{c}n+1 \\ 2\end{array}\right)$. With this in mind, define (idempotent) partitions $\bar{\varepsilon}_{i}(1 \leq i \leq n)$ and $\bar{t}_{i j}(1 \leq i<j \leq n)$ as in Figure 7 .
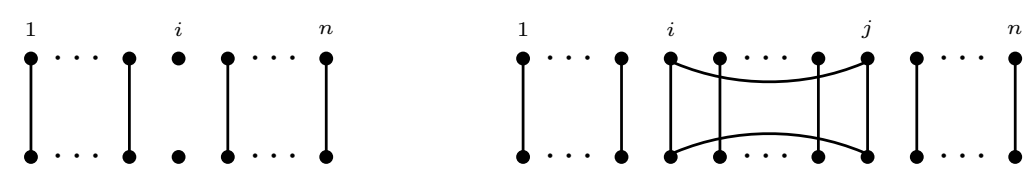

Figure 7: The partitions $\bar{\varepsilon}_{i}$ (left) and $\bar{t}_{i j}$ (right).

Proposition 16 The semigroup $\mathcal{P}_{n} \backslash \mathcal{S}_{n}$ is generated by the set

$$
\Sigma=\left\{\bar{\varepsilon}_{i} \mid 1 \leq i \leq n\right\} \cup\left\{\bar{t}_{i j} \mid 1 \leq i<j \leq n\right\} .
$$

Proof It suffices to show that $\overline{\mathscr{X}}$ is contained in $\langle\Sigma\rangle$, the subsemigroup of $\mathcal{P}_{n} \backslash \mathcal{S}_{n}$ generated by $\Sigma$. Now $\bar{\lambda}_{n}=\bar{\varepsilon}_{n}$ and, as may be easily checked diagrammatically, we have $\bar{\lambda}_{i}=\left(\bar{\varepsilon}_{i} \bar{t}_{i, i+1}\right) \bar{\lambda}_{i+1}$ for all $1 \leq i \leq n-1$. Inductively, this gives rise to the expression

$$
\bar{\lambda}_{i}=\bar{\varepsilon}_{i} \bar{t}_{i, i+1} \bar{\varepsilon}_{i+1} \bar{t}_{i+1, i+2} \cdots \bar{\varepsilon}_{n-1} \bar{t}_{n-1, n} \bar{\varepsilon}_{n} .
$$

By applying the anti-involution ${ }^{\circ}: \mathcal{P}_{n} \rightarrow \mathcal{P}_{n}$, it follows that

$$
\bar{\rho}_{i}=\bar{\varepsilon}_{n} \bar{t}_{n-1, n} \bar{\varepsilon}_{n-1} \cdots \bar{t}_{i+1, i+2} \bar{\varepsilon}_{i+1} \bar{t}_{i, i+1} \bar{\varepsilon}_{i} .
$$

One may also verify that

$$
\bar{\alpha}_{i j}=\bar{t}_{i j} \bar{\lambda}_{j}, \quad \bar{\beta}_{i j}=\bar{\rho}_{j} \bar{t}_{i j}, \quad \bar{s}_{r}=\bar{\varepsilon}_{n} \bar{t}_{r, n} \bar{\varepsilon}_{r} \bar{t}_{r, r+1} \bar{\varepsilon}_{r+1} \bar{t}_{r+1, n} \bar{\varepsilon}_{n},
$$

and the proof is complete. 
Remark 17 In the reverse direction, the new generators can be expressed in terms of the old as

$$
\bar{\varepsilon}_{i}=\bar{\lambda}_{i} \bar{\rho}_{i} \quad \text { and } \quad \bar{t}_{i j}=\bar{\alpha}_{i j} \bar{\beta}_{i j}
$$

Remark 18 The subsets $\left\{\bar{\varepsilon}_{i} \mid 1 \leq i \leq n\right\}$ and $\left\{\bar{t}_{i j} \mid 1 \leq i<j \leq n\right\}$ respectively generate (as monoids) $E\left(\mathcal{I}_{n}\right)$ and $E\left(\mathcal{I}_{n}^{*}\right)$, the idempotent semilattices of the symmetric and dual symmetric inverse monoids (see $[3,4,10]$. So this proposition also shows that

$$
\left(\mathcal{P}_{n} \backslash \mathcal{S}_{n}\right) \cup\{1\}=\left\langle E\left(\mathcal{P}_{n}\right)\right\rangle=\left\langle E\left(\mathcal{I}_{n}\right) \cup E\left(\mathcal{I}_{n}^{*}\right)\right\rangle
$$

Theorem 19 The semigroup $\mathcal{P}_{n} \backslash \mathcal{S}_{n}$ is generated by its idempotents, and we have

$$
\operatorname{rank}\left(\mathcal{P}_{n} \backslash \mathcal{S}_{n}\right)=\operatorname{id}-\operatorname{rank}\left(\mathcal{P}_{n} \backslash \mathcal{S}_{n}\right)=\left(\begin{array}{c}
n+1 \\
2
\end{array}\right)=\frac{n(n+1)}{2}
$$

Proof Since every element of $\Sigma$ is idempotent it suffices, by Proposition 16, to show that $\operatorname{rank}\left(\mathcal{P}_{n} \backslash \mathcal{S}_{n}\right) \geq\left(\begin{array}{c}n+1 \\ 2\end{array}\right)$. With this in mind, let $\Gamma$ be an arbitrary generating set for $\mathcal{P}_{n} \backslash \mathcal{S}_{n}$. We must show that $|\Gamma| \geq\left(\begin{array}{c}n+1 \\ 2\end{array}\right)$.

Let $i \in \mathbf{n}$, and consider an expression $\bar{\varepsilon}_{i}=\gamma_{1} \cdots \gamma_{k}$ where $\gamma_{1}, \ldots, \gamma_{k} \in \Gamma$. We wish to show that $\operatorname{dom}\left(\gamma_{1}\right)=\mathbf{n} \backslash\{i\}$, so suppose for the moment that this is not the case. Now

$$
\mathbf{n} \backslash\{i\}=\operatorname{dom}\left(\bar{\varepsilon}_{i}\right)=\operatorname{dom}\left(\gamma_{1} \cdots \gamma_{k}\right) \subseteq \operatorname{dom}\left(\gamma_{1}\right),
$$

and it follows (by assumption) that $\operatorname{dom}\left(\gamma_{1}\right)=\mathbf{n}$. We also have

$$
\Delta=\operatorname{ker}\left(\bar{\varepsilon}_{i}\right)=\operatorname{ker}\left(\gamma_{1} \cdots \gamma_{k}\right) \supseteq \operatorname{ker}\left(\gamma_{1}\right),
$$

so that $\operatorname{ker}\left(\gamma_{1}\right)=\Delta$. Together with $\operatorname{dom}\left(\gamma_{1}\right)=\mathbf{n}$, this implies that $\gamma_{1} \in \mathcal{S}_{n}$, contradicting the fact that $\gamma_{1} \in \mathcal{P}_{n} \backslash \mathcal{S}_{n}$. It follows that $\operatorname{dom}\left(\gamma_{1}\right)=\mathbf{n} \backslash\{i\}$. Repeating this argument for all $i \in \mathbf{n}$, we see that $\Gamma$ contains (at least) $n$ distinct partitions with domain a proper subset of $\mathbf{n}$.

Now let $1 \leq i<j \leq n$, and consider an expression $\bar{t}_{i j}=\eta_{1} \cdots \eta_{k}$ where $\eta_{1}, \ldots, \eta_{k} \in \Gamma$. Note that

$$
\mathbf{n}=\operatorname{dom}\left(\bar{t}_{i j}\right)=\operatorname{dom}\left(\eta_{1} \cdots \eta_{k}\right) \subseteq \operatorname{dom}\left(\eta_{1}\right),
$$

so that $\operatorname{dom}\left(\eta_{1}\right)=\mathbf{n}$. Write $\mathscr{E}_{i j}$ for the equivalence $\Delta \cup\{(i, j),(j, i)\}$. Now

$$
\mathscr{E}_{i j}=\operatorname{ker}\left(\bar{t}_{i j}\right)=\operatorname{ker}\left(\eta_{1} \cdots \eta_{k}\right) \supseteq \operatorname{ker}\left(\eta_{1}\right),
$$

so either $\operatorname{ker}\left(\eta_{1}\right)=\Delta$ or $\operatorname{ker}\left(\eta_{1}\right)=\mathscr{E}_{i j}$. But since $\operatorname{dom}\left(\eta_{1}\right)=\mathbf{n}$ and $\eta_{1} \notin \mathcal{S}_{n}$, we cannot have $\operatorname{ker}\left(\eta_{1}\right)=\Delta$, so it follows that $\operatorname{ker}\left(\eta_{1}\right)=\mathscr{E}_{i j}$. Repeating this argument for all $1 \leq i<j \leq n$, we see that $\Gamma$ contains (at least) $\left(\begin{array}{l}n \\ 2\end{array}\right)$ distinct partitions with domain equal to $\mathbf{n}$.

Putting together the conclusions of the previous two paragraphs, we have

$$
|\Gamma| \geq n+\left(\begin{array}{l}
n \\
2
\end{array}\right)=\left(\begin{array}{c}
n+1 \\
2
\end{array}\right)
$$

and the proof is complete. 
Remark 20 Dual arguments to those in the above proof show that any generating set $\Gamma$ of $\mathcal{P}_{n} \backslash \mathcal{S}_{n}$ must contain an element with codomain $\mathbf{n} \backslash\{i\}$ and cokernel $\Delta$ for each $i$, and an element with cokernel $\mathscr{E}_{i j}$ for each $i, j$. This shows that the generating set

$$
\left\{\bar{\alpha}_{i j} \mid 1 \leq i<j \leq n\right\} \cup\left\{\bar{\beta}_{i j} \mid 1 \leq i<j \leq n\right\} \cup\left\{\bar{\lambda}_{i} \mid 1 \leq i \leq n-1\right\} \cup\left\{\bar{\rho}_{i} \mid 1 \leq i \leq n-1\right\}
$$

discussed in Remark 15 cannot be reduced further, although it is not of minimal cardinality.

Remark 21 The set $\Sigma$ is not the only idempotent generating set of minimal rank. The characterization of such minimal idempotent generating sets is the subject of another work [5].

\section{Refining the Presentation}

We now set out to find a presentation for $\mathcal{P}_{n} \backslash \mathcal{S}_{n}$ in terms of the idempotent generating set $\Sigma$ from Proposition 16. To do this, we start with the presentation $\langle\mathscr{X} \mid \mathscr{R}\rangle$ from Theorem 14, and perform a series of Tietze transformations. With this in mind, define an alphabet

$$
\mathscr{Y}=\left\{\varepsilon_{i} \mid 1 \leq i \leq n\right\} \cup\left\{t_{i j} \mid 1 \leq i<j \leq n\right\} .
$$

Beginning with $\langle\mathscr{X} \mid \mathscr{R}\rangle$, we add the generators from $\mathscr{Y}$, along with the relations

$$
\begin{aligned}
\varepsilon_{i} & =\lambda_{i} \rho_{i} & & \text { for all } i \\
t_{i j} & =\alpha_{i j} \beta_{i j} & & \text { for all } i, j,
\end{aligned}
$$

which define them in terms of the original generators. Writing $\mathscr{D}$ for the set of relations (D1-D2), the presentation becomes $\langle\mathscr{X} \cup \mathscr{Y} \mid \mathscr{R} \cup \mathscr{D}\rangle$. In the statement of the next lemma, and indeed for the duration of this article, we will use the symmetric notation $t_{j i}=t_{i j}$ for all $1 \leq i<j \leq n$ (think of both expressions standing for the slightly more clumsy $\left.t_{\{i, j\}}\right)$.

Lemma 22 With $i, j, k, l$ ranging over all possible indices, subject to the stated constraints, the following relations are in $(\mathscr{R} \cup \mathscr{D})^{\sharp}$ :

$$
\begin{aligned}
\varepsilon_{i}^{2} & =\varepsilon_{i} \\
\varepsilon_{i} \varepsilon_{j} & =\varepsilon_{j} \varepsilon_{i} \\
t_{i j}^{2} & =t_{i j} \\
t_{i j} t_{k l} & =t_{k l} t_{i j} \\
t_{i j} t_{j k}=t_{j k} t_{k i} & =t_{k i} t_{i j} \\
t_{i j} \varepsilon_{k} & =\varepsilon_{k} t_{i j} \\
t_{i j} \varepsilon_{k} t_{i j} & =t_{i j} \\
\varepsilon_{k} t_{i j} \varepsilon_{k} & =\varepsilon_{k} \\
\varepsilon_{k} t_{k i} \varepsilon_{i} t_{i j} \varepsilon_{j} t_{j k} \varepsilon_{k} & =\varepsilon_{k} t_{k j} \varepsilon_{j} t_{j i} \varepsilon_{i} t_{i k} \varepsilon_{k} \\
\varepsilon_{k} t_{k i} \varepsilon_{i} t_{i j} \varepsilon_{j} t_{j l} \varepsilon_{l} t_{l k} \varepsilon_{k} & =\varepsilon_{k} t_{k l} \varepsilon_{l} t_{l i} \varepsilon_{i} t_{i j} \varepsilon_{j} t_{j k} \varepsilon_{k} .
\end{aligned}
$$


Proof This follows from a simple diagrammatic check, and the fact that $\langle\mathscr{X} \cup \mathscr{Y} \mid \mathscr{R} \cup \mathscr{D}\rangle$ is a presentation.

Denote by $\mathscr{Q}$ the set of relations in the statement of Lemma 22 . We add these relations to the presentation to obtain

$$
\langle\mathscr{X} \cup \mathscr{Y} \mid \mathscr{R} \cup \mathscr{D} \cup \mathscr{Q}\rangle .
$$

It is our ultimate goal to show that the presentation simplifies to $\langle\mathscr{Y} \mid \mathscr{Q}\rangle$. Throughout our pursuit of this goal, certain simple words (and longer words built up out of them) will play a crucial role. For a pair of distinct indices $i, j \in \mathbf{n}$, we define

$$
X_{i j}=\varepsilon_{i} t_{i j} \varepsilon_{j} \in \mathscr{Y}^{+} .
$$

As always, we are using symmetric notation for the $t_{i j}=t_{j i}$. However, it is important to note that $X_{j i} \neq X_{i j}$. We further define words

$$
\begin{aligned}
& L_{i}= \begin{cases}X_{i, i+1} X_{i+1, i+2} \cdots X_{n-1, n} & \text { for } 1 \leq i \leq n-1 \\
\varepsilon_{n} & \text { for } i=n\end{cases} \\
& R_{i}= \begin{cases}X_{n, n-1} \cdots X_{i+2, i+1} X_{i+1, i} & \text { for } 1 \leq i \leq n-1 \\
\varepsilon_{n} & \text { for } i=n\end{cases} \\
& A_{i j}=t_{i j} L_{j} \quad \text { for } 1 \leq i<j \leq n \\
& B_{i j}=R_{j} t_{i j} \quad \text { for } 1 \leq i<j \leq n \\
& S_{r}=X_{n r} X_{r, r+1} X_{r+1, n} \quad \text { for } 1 \leq r \leq n-2 .
\end{aligned}
$$

As the notation suggests, these words over $\mathscr{Y}$ map to the generators $\bar{\lambda}_{i}, \bar{\rho}_{i}$, etc., of $\mathcal{P}_{n} \backslash \mathcal{S}_{n}$, as made precise in the statement of the next lemma.

Lemma 23 The following relations are in $(\mathscr{R} \cup \mathscr{D} \cup \mathscr{Q})^{\sharp}:$

$$
\begin{array}{rlr}
\lambda_{i}=L_{i}, \quad \rho_{i}=R_{i} & \text { for all } i \\
\alpha_{i j}=A_{i j}, \quad \beta_{i j}=B_{i j} & \text { for all } i, j \\
s_{r}=S_{r} & \text { for all } r .
\end{array}
$$

Proof Again a simple diagrammatic check is sufficient, since $\langle\mathscr{X} \cup \mathscr{Y} \mid \mathscr{R} \cup \mathscr{D} \cup \mathscr{Q}\rangle$ is known to be a presentation.

As a result, the generators from $\mathscr{X}$ may be removed from the presentation, with their every occurrence in the relations $\mathscr{R} \cup \mathscr{D}$ being replaced by the words $L_{i}, R_{i}, A_{i j}, B_{i j}, S_{r}$ as appropriate. We denote the resulting relations by (L1)', etc., and the entire set of modified relations by $\mathscr{R}^{\prime} \cup \mathscr{D}^{\prime}$. The presentation has now become

$$
\left\langle\mathscr{Y} \mid \mathscr{R}^{\prime} \cup \mathscr{D}^{\prime} \cup \mathscr{Q}\right\rangle,
$$


and our goal has become to show that relations $\mathscr{R}^{\prime} \cup \mathscr{D}^{\prime}$ follow from $\mathscr{Q}$. For the remainder of the article, we will write $\approx=\mathscr{Q}^{\sharp}$ for the congruence on $\mathscr{Y}^{+}$generated by $\mathscr{Q}$.

We will be aided in our goal by a duality in the relations. Define the anti-involution

$$
\widehat{\imath} \mathscr{Y}^{+} \rightarrow \mathscr{Y}^{+}
$$

to be the extension of the identity map $\mathscr{Y} \rightarrow \mathscr{Y}$.

Lemma 24 Let $w_{1}, w_{2} \in \mathscr{Y}^{+}$. Then $w_{1} \approx w_{2}$ if and only if $\widehat{w}_{1} \approx \widehat{w}_{2}$.

Proof This follows immediately from the fact that $\mathscr{Q}$ is closed under $\widehat{ }$.

Of special importance is the fact that $\widehat{X}_{i j}=X_{j i}$, from which it follows that

$$
\widehat{L}_{i}=R_{i}, \quad \widehat{R}_{i}=L_{i}, \quad \widehat{A}_{i j}=B_{i j}, \quad \widehat{B}_{i j}=A_{i j} .
$$

We will see later that $\widehat{S}_{r} \approx S_{r}$ also.

Before considering the relations from $\mathscr{R}^{\prime} \cup \mathscr{D}^{\prime}$ one-by-one, we first prove some results concerning the words $X_{i j}=\varepsilon_{i} t_{i j} \varepsilon_{j}$ defined above.

Lemma 25 With $i, j, k, l$ ranging over all possible indices, subject to the stated constraints, we have:

(25.1) $X_{i j}^{3} \approx X_{i j}^{2} \approx \varepsilon_{i} \varepsilon_{j}$;

(25.2) $X_{i j} X_{k l} \approx X_{k l} X_{i j}$, if $\{i, j\} \cap\{k, l\}=\emptyset$;

(25.3) $X_{i j}\left(X_{j k} X_{i j}\right) \approx X_{j i}\left(X_{j k} X_{i j}\right) \approx\left(X_{j k} X_{i j}\right) X_{j k} \approx\left(X_{j k} X_{i j}\right) X_{k j} \approx X_{j k} X_{i j}$, if $i \neq k$;

(25.4) $\left(X_{i j} X_{j k}\right) X_{j i} \approx X_{k j}\left(X_{i j} X_{j k}\right) \approx \varepsilon_{i} \varepsilon_{k}$, if $i \neq k$; and

(25.5) $X_{i j} X_{j i} \approx \varepsilon_{i}$.

Proof First we have

$$
X_{i j}^{2}=\varepsilon_{i} t_{i j} \varepsilon_{j} \varepsilon_{i} t_{i j} \varepsilon_{j} \approx \varepsilon_{i} t_{i j} \varepsilon_{i} \varepsilon_{j} t_{i j} \varepsilon_{j} \approx \varepsilon_{i} \varepsilon_{j},
$$

by (E2) and (ET3), while

$$
X_{i j}^{3} \approx \varepsilon_{i} \varepsilon_{j} X_{i j}=\varepsilon_{i} \varepsilon_{j} \varepsilon_{i} t_{i j} \varepsilon_{j} \sim \varepsilon_{i} \varepsilon_{i} \varepsilon_{j} t_{i j} \varepsilon_{j} \sim \varepsilon_{i} \varepsilon_{j},
$$

by (E1-E2), (ET3), and the previous calculation, completing the proof of (25.1).

Relation (25.2) is an immediate consequence of (E2), (T2), and (ET1). 
For (25.3), note that if $i, j, k$ are distinct, then

$$
\begin{aligned}
X_{j k} X_{i j}=\varepsilon_{j} t_{j k} \varepsilon_{k} \varepsilon_{i} t_{i j} \varepsilon_{j} & \approx \varepsilon_{i} \varepsilon_{j} t_{j k} t_{i j} \varepsilon_{j} \varepsilon_{k} & & \text { by (E2) and (ET1) } \\
& \approx X_{i j}^{2} t_{j k} t_{i j} X_{j k}^{2} & & \text { by (25.1) above } \\
& \approx X_{j i}^{2} t_{j k} t_{i j} X_{k j}^{2} & & \text { by (25.1) again. }
\end{aligned}
$$

By another application of (25.1) we see that, modulo the relations, $X_{j k} X_{i j}$ acts as a rightzero for $X_{i j}$ and $X_{j i}$, and as a left-zero for $X_{j k}$ and $X_{k j}$.

Next, with $i, j, k$ distinct again, we have

$$
\begin{aligned}
\left(X_{i j} X_{j k}\right) X_{j i} & =\varepsilon_{i} t_{i j} \varepsilon_{j} \varepsilon_{j} t_{j k} \varepsilon_{k} \varepsilon_{j} t_{j i} \varepsilon_{i} & & \\
& \approx \varepsilon_{i} t_{i j} \varepsilon_{j} t_{j k} \varepsilon_{j} t_{j i} \varepsilon_{i} \varepsilon_{k} & & \text { by (E1) and (ET1) } \\
& \approx \varepsilon_{i} t_{i j} \varepsilon_{j} t_{j i} \varepsilon_{i} \varepsilon_{k} & & \text { by (ET3) } \\
& \approx \varepsilon_{i} t_{i j} \varepsilon_{i} \varepsilon_{k} & & \text { by (ET2) } \\
& \approx \varepsilon_{i} \varepsilon_{k} & & \text { by (ET3) },
\end{aligned}
$$

establishing the first part of (25.4). The second part follows from the first by duality (i.e. an application of Lemma 24) and relation (E2).

Finally, (25.5) follows quickly from (E1), (ET2), and (ET3).

We now begin to eliminate the relations from $\mathscr{D}^{\prime} \cup \mathscr{R}^{\prime}$. The order in which we proceed is determined partly by difficulty, but also by convenience in terms of using established relations in the subsequent proofs of other relations.

Lemma 26 Relations (D1-D2)' are in $\mathscr{Q}^{\sharp}$.

Proof For (D1)' we must prove that $\varepsilon_{i} \approx L_{i} R_{i}$. We do this by backwards induction on $i$. Now if $i=n$, then we are done by (E1), since $L_{n}=R_{n}=\varepsilon_{n}$, so suppose $1 \leq i \leq n-1$. Note that

$$
L_{i} \approx X_{i, i+1} L_{i+1} \quad \text { and } \quad R_{i} \approx R_{i+1} X_{i+1, i} .
$$

(Indeed, we have equality in the case $1 \leq i \leq n-2$, but must apply (E1) in the case $i=n-1$.) It then follows that

$$
L_{i} R_{i} \approx X_{i, i+1} L_{i+1} R_{i+1} X_{i+1, i} \approx X_{i, i+1} \varepsilon_{i+1} X_{i+1, i} \approx X_{i, i+1} X_{i+1, i} \approx \varepsilon_{i},
$$

where we have used an induction hypothesis, as well as (E1) and (25.5).

For $(\mathrm{D} 2)^{\prime}$, we have

$$
A_{i j} B_{i j}=t_{i j} L_{j} R_{j} t_{i j} \approx t_{i j} \varepsilon_{j} t_{i j} \approx t_{i j}
$$

where we have applied (D1)' and (ET2). 
Lemma 27 Relations $(\mathrm{L} 1-\mathrm{L} 2)^{\prime}$ and $(\mathrm{R} 1-\mathrm{R} 2)^{\prime}$ are in $\mathscr{Q}^{\sharp}$.

Proof Relation (L2) follows immediately from (E1). For $(\mathrm{L} 1)^{\prime}$, it will be convenient for the moment to use the notation $x_{i}=X_{i, i+1}$ for $i=1, \ldots, n-1$. By (25.2) and (25.3) the following (braid) relations hold:

$$
\begin{aligned}
x_{i} x_{j} & \approx x_{j} x_{i} & & \text { if }|i-j|>1 \\
x_{i} x_{i+1} x_{i} & \approx x_{i+1} x_{i} x_{i+1} & & \text { if } 1 \leq i \leq n-2 .
\end{aligned}
$$

If we further define $x_{n}=\varepsilon_{n}$, then one may easily check that (27.1) and (27.2) above also hold when the indices are allowed to be $n$. Now, using (27.1) and (27.2), observe that if $1 \leq i \leq k \leq n-1$, then

$$
\begin{gathered}
L_{i} x_{k} \approx x_{i} \cdots x_{k-1} x_{k} x_{k+1} x_{k+2} \cdots x_{n-1} x_{k} \approx x_{i} \cdots x_{k-1} x_{k} x_{k+1} x_{k} x_{k+2} \cdots x_{n-1} \\
\approx x_{i} \cdots x_{k-1} x_{k+1} x_{k} x_{k+1} x_{k+2} \cdots x_{n-1} \approx x_{k+1} x_{i} \cdots x_{k-1} x_{k} x_{k+1} x_{k+2} \cdots x_{n-1}=x_{k+1} L_{i} .
\end{gathered}
$$

(Here, note that the first equivalence is an equality unless $k=n-1$, in which case the subword $x_{k+2} \cdots x_{n-1}$ is assumed to be empty.) So, for $1 \leq i \leq j \leq n-1$, we have

$$
L_{i} L_{j}=L_{i} x_{j} \cdots x_{n-1} \approx x_{j+1} \cdots x_{n} L_{i} \approx L_{j+1} L_{n} L_{i} \approx L_{j+1} L_{i},
$$

by the above observation and (L2)', completing the proof of (L1)'. (Again, the second equivalence is an equality unless $j=n-1$.)

Finally, relations $(\mathrm{R} 1-\mathrm{R} 2)^{\prime}$ follow from $(\mathrm{L} 1-\mathrm{L} 2)^{\prime}$ and duality.

Lemma 28 Relations (RL1-RL3)' are in $\mathscr{Q}^{\sharp}$.

Proof For $(\mathrm{RL} 2)^{\prime}$, note first that $R_{n} L_{n}=\varepsilon_{n}^{2} \approx \varepsilon_{n}=L_{n}$ by (E1), while if $1 \leq i \leq n-1$, then

$$
R_{i} L_{i} \approx R_{i+1} X_{i+1, i} X_{i, i+1} L_{i+1} \approx R_{i+1} \varepsilon_{i+1} L_{i+1} \approx R_{i+1} L_{i+1} \approx \varepsilon_{n}=L_{n},
$$

where we have used (25.5), (E1), and an induction hypothesis.

We now consider (RL1)'. Again it will be convenient to use the notation $x_{i}(i \in \mathbf{n})$ defined in the proof of the previous lemma. We further define $y_{i}=\widehat{x}_{i}$ for each $i$; that is, $y_{i}=X_{i+1, i}$ for $i=1, \ldots, n-1$ and $y_{n}=\varepsilon_{n}$. By (24.2) and (24.4) the following (singular braid) relations hold:

$$
\begin{aligned}
x_{i} y_{j} & \approx y_{j} x_{i} & & \text { if }|i-j|>1 \\
x_{i} y_{i+1} y_{i} & \approx y_{i+1} y_{i} x_{i+1} & & \text { if } 1 \leq i \leq n-2 .
\end{aligned}
$$

Using these, a similar calculation yields

$$
R_{i} x_{k} \approx x_{k-1} R_{i} \quad \text { if } 1 \leq i<k \leq n-1 .
$$


We also calculate

$$
x_{n} x_{n-1}=\varepsilon_{n} \varepsilon_{n-1} t_{n-1, n} \varepsilon_{n} \approx \varepsilon_{n-1} \varepsilon_{n} t_{n-1, n} \varepsilon_{n} \approx \varepsilon_{n-1} \varepsilon_{n}
$$

by (E2) and (ET3). Putting this together, for $1 \leq i<j \leq n-1$, we have

$$
\begin{aligned}
R_{i} L_{j}=R_{i} x_{j} \cdots x_{n-1} & \approx x_{j-1} \cdots x_{n-2} R_{i} & & \text { by }(28.3) \\
& \approx x_{j-1} \cdots x_{n-2} \varepsilon_{n-1} \varepsilon_{n} R_{i} & & \text { by }(\mathrm{E} 1) \\
& \approx x_{j-1} \cdots x_{n-2} x_{n} x_{n-1} R_{i} & & \text { by }(28.4) \\
& \approx x_{n} x_{j-1} \cdots x_{n-2} x_{n-1} R_{i} & & \text { by }(27.1) \\
& =L_{n} L_{j-1} R_{i} . & &
\end{aligned}
$$

Finally, for the $j=n$ case of (RL1)', note first that by (28.4) above, and duality, we have

$$
R_{n-1} R_{n}=y_{n-1} y_{n} \approx \varepsilon_{n-1} \varepsilon_{n} \approx x_{n} x_{n-1}=L_{n} L_{n-1} .
$$

By $(\mathrm{R} 1-\mathrm{R} 2)^{\prime}$ and $(28.5)$, if $1 \leq i<n$, then

$$
R_{i} L_{n}=R_{i} R_{n} \approx R_{n-1} R_{i} \approx R_{n-1} R_{n} R_{i} \approx L_{n} L_{n-1} R_{i},
$$

completing the proof of $(\mathrm{RL} 1)^{\prime}$. For $(\mathrm{RL} 3)^{\prime}$, suppose $1 \leq j<i \leq n$. Then

$$
\begin{aligned}
R_{j} L_{i} & \approx L_{n} L_{i-1} R_{j} & & \text { by }(\mathrm{RL} 1)^{\prime} \\
& \approx L_{i-1} L_{n-1} R_{j} & & \text { by }(\mathrm{L} 1)^{\prime} \\
& \approx L_{i-1} L_{n} L_{n-1} R_{j} & & \text { by }(\mathrm{L} 2)^{\prime} \\
& \approx L_{i-1} R_{n-1} R_{n} R_{j} & & \text { by }(28.5) \\
& \approx L_{i-1} R_{n-1} R_{j} & & \text { by }(\mathrm{R} 2)^{\prime} \\
& \approx L_{i-1} R_{j} R_{n} & & \text { by }(\mathrm{R} 1)^{\prime} .
\end{aligned}
$$

By duality it follows that $R_{i} L_{j} \approx L_{n} L_{j} R_{i-1}$, and the proof is complete.

In order to deal with relations $(\mathrm{A} 1-\mathrm{A} 5)^{\prime}$ and $(\mathrm{B} 1-\mathrm{B} 5)^{\prime}$, we first need some knowledge of how the words $X_{k l}$ and $L_{k}$ interact with the generators $t_{i j}$.

Lemma 29 Let $i, j, k, l \in \mathbf{n}$ with $i \neq j$ and $k \neq l$. Then

$$
X_{k l} t_{i j} \approx \begin{cases}t_{i j} X_{k l} & \text { if }\{i, j\} \cap\{k, l\}=\emptyset \\ t_{j l} X_{k l} & \text { if } k=i \text { but } l \neq j \\ \varepsilon_{i} t_{i j} & \text { if } k=i \text { and } l=j .\end{cases}
$$

(Note that not all "cases" have been considered.) 
Proof Relation (29.1) follows immediately from (ET1) and (T2). For (29.2) we have

$$
X_{k l} t_{k j}=\varepsilon_{k} t_{k l} \varepsilon_{l} t_{k j} \approx \varepsilon_{k} t_{k l} t_{k j} \varepsilon_{l} \approx \varepsilon_{k} t_{j l} t_{k l} \varepsilon_{l} \approx t_{j l} \varepsilon_{k} t_{k l} \varepsilon_{l}=t_{j l} X_{k l},
$$

using (ET1) and (T3). Finally, for (29.3) we have

$$
X_{i j} t_{i j}=\varepsilon_{i} t_{i j} \varepsilon_{j} t_{i j} \approx \varepsilon_{i} t_{i j}
$$

by (ET2), and the proof is complete.

Lemma 30 Let $1 \leq k \leq n$ and $1 \leq i<j \leq n-1$. Then

$$
L_{k} t_{i j} \approx \begin{cases}t_{i j} L_{k} & \text { if } j<k \\ t_{i, j+1} L_{k} & \text { if } i<k \leq j \\ t_{i+1, j+1} L_{k} & \text { if } k \leq i,\end{cases}
$$

and

$$
t_{i j} R_{k} \approx \begin{cases}R_{k} t_{i j} & \text { if } j<k \\ R_{k} t_{i, j+1} & \text { if } i<k \leq j \\ R_{k} t_{i+1, j+1} & \text { if } k \leq i\end{cases}
$$

Proof Relation (30.1) follows from (29.1) if $k<n$, or (ET1) if $k=n$. For (30.2) we have

$$
\begin{array}{rlr}
L_{k} t_{i j} & =X_{k, k+1} \cdots X_{j-1, j} X_{j, j+1} X_{j+1, j+2} \cdots X_{n-1, n} t_{i j} & \\
& \approx X_{k, k+1} \cdots X_{j-1, j} X_{j, j+1} t_{i j} X_{j+1, j+2} \cdots X_{n-1, n} & \text { by }(29.1) \\
& \approx X_{k, k+1} \cdots X_{j-1, j} t_{i, j+1} X_{j, j+1} X_{j+1, j+2} \cdots X_{n-1, n} & \text { by }(29.2) \\
& \approx t_{i, j+1} X_{k, k+1} \cdots X_{j-1, j} X_{j, j+1} X_{j+1, j+2} \cdots X_{n-1, n} & \text { by }(29.1) \\
& =t_{i, j+1} L_{k} . &
\end{array}
$$

Relation (30.3) follows by a similar calculation, and (30.4-30.6) follow by duality.

Lemma 31 Relations $(\mathrm{A} 1-\mathrm{A} 5)^{\prime}$ and $(\mathrm{B} 1-\mathrm{B} 5)^{\prime}$ are in $\mathscr{Q}^{\sharp}$.

Proof For $(\mathrm{A} 1)^{\prime}$, we use (L2)' and (ET3), noting that $L_{n}=\varepsilon_{n}$, to obtain

$$
A_{k l} A_{i j}=t_{k l} L_{l} t_{i n} L_{n} \approx t_{k l} L_{l} L_{n} t_{i n} L_{n}=t_{k l} L_{l} L_{n} \approx t_{k l} L_{l}=A_{k l} .
$$

For (A2)', suppose $1 \leq i<j<k \leq n$. Then by (30.1) and (T2-T3) we have

$$
A_{j k} A_{i j}=t_{j k} L_{k} t_{i j} L_{j} \approx t_{j k} t_{i j} L_{k} L_{j} \approx t_{i k} t_{i j} L_{k} L_{j} \approx t_{i k} L_{k} t_{i j} L_{j}=A_{i k} A_{i j} .
$$

Also, using one of the intermediate stages of the previous calculation, as well as (T2), (L1)', and (30.2), we calculate

$$
A_{j k} A_{i j} \approx t_{i k} t_{i j} L_{k} L_{j} \approx t_{i j} t_{i k} L_{j} L_{k-1} \approx t_{i j} L_{j} t_{i, k-1} L_{k-1}=A_{i j} A_{i, k-1} .
$$

Relations (A3-A5)' are proved in a similar fashion. Relations (B1-B5)' follow by duality. 
Lemma 32 Relations (LA1-LA4)' and (BR1-BR4)' are in $\mathscr{Q}^{\sharp}$.

Proof The proof of $(\mathrm{LA} 1)^{\prime}$ is similar to that of $(\mathrm{A} 1)^{\prime}$. For $(\mathrm{LA} 2)^{\prime}$, if $1 \leq k \leq i<j<n$, then

$$
L_{k} A_{i j}=L_{k} t_{i j} L_{j} \approx t_{i+1, j+1} L_{k} L_{j} \approx t_{i+1, j+1} L_{j+1} L_{k}=A_{i+1, j+1} L_{k},
$$

by (30.3) and (L1)'. Relations (LA3-LA4)' are proved in an almost identical manner. Relations (BR1-BR4)' follow by duality.

Lemma 33 Relations $(\mathrm{AL} 1)^{\prime}$ and $(\mathrm{RB} 1)^{\prime}$ are in $\mathscr{Q}^{\sharp}$.

Proof By $(\mathrm{L} 2)^{\prime}$ we have

$$
A_{i j} L_{n}=t_{i j} L_{j} L_{n} \approx t_{i j} L_{j}=A_{i j}
$$

establishing (AL1)'. Relation (RB1)' follows by duality.

Lemma 34 Relations (BA1-BA9)' are in $\mathscr{Q}^{\sharp}$.

Proof For $(\mathrm{BA} 1)^{\prime}$, suppose $1 \leq k<l<i<j \leq n$. Then

$$
\begin{aligned}
B_{k l} A_{i j} & =R_{l} t_{k l} t_{i j} L_{j} & & \\
& \approx R_{l} t_{i j} t_{k l} L_{j} & & \text { by }(\mathrm{T} 2) \\
& \approx t_{i-1, j-1} R_{l} L_{j} t_{k l} & & \text { by }(30.1) \text { and }(30.6) \\
& \approx t_{i-1, j-1} L_{n} L_{j-1} R_{l} t_{k l} & & \text { by }(\mathrm{RL} 1)^{\prime} \\
& \approx L_{n} t_{i-1, j-1} L_{j-1} R_{l} t_{k l} & & \text { by }(30.1) \\
& =L_{n} A_{i-1, j-1} B_{k l} . & &
\end{aligned}
$$

With the exception of $(\mathrm{BA} 5)^{\prime}$, the remaining relations are dealt with in an essentially identical manner. For $(\mathrm{BA} 5)^{\prime}$ we have

$$
B_{i j} A_{i j}=R_{j} t_{i j} t_{i j} L_{j} \approx R_{j} \varepsilon_{j} t_{i j} \varepsilon_{j} L_{j} \approx R_{j} \varepsilon_{j} L_{j} \approx R_{j} L_{j} \approx L_{n}
$$

where we have used (T1), (E1), (ET3), and (RL2)'.

Before moving on to the relations that involve the $S_{i}$, we must prove some more technical results concerning the words $X_{i j}$.

Lemma 35 Let $i, j, k \in \mathbf{n}$ be distinct. Then

$$
X_{i k} X_{j k} \approx X_{i j} X_{i k} \approx X_{j k} X_{i j} \approx \varepsilon_{j} X_{i k} \approx X_{i k} \varepsilon_{j} .
$$


Proof By (E2), (ET1), and (ET3), we have

$$
X_{i k} X_{j k}=\varepsilon_{i} t_{i k} \varepsilon_{k} \varepsilon_{j} t_{j k} \varepsilon_{k} \approx \varepsilon_{j} \varepsilon_{i} t_{i k} \varepsilon_{k} t_{j k} \varepsilon_{k} \approx \varepsilon_{j} \varepsilon_{i} t_{i k} \varepsilon_{k}=\varepsilon_{j} X_{i k} .
$$

The other relations are established analogously.

It will also help to know that $\widehat{S}_{r} \approx S_{r}$ for all $r$, so that we will be able to apply duality arguments to relations involving the $S_{r}$. This is a special case of the following lemma which, incidentally, will necessitate our first use of relation (ET4). For distinct integers $i, j, k \in \mathbf{n}$, we define a word

$$
S_{i j ; k}=X_{k i} X_{i j} X_{j k}
$$

Note in particular that $S_{r}=S_{r, r+1 ; n}$ for all $r$. One may easily check that the word $S_{i j ; k}$ is mapped to the restriction of transposition $(i, j)$ to the domain $\mathbf{n} \backslash\{k\}$.

Lemma 36 Let $i, j, k \in \mathbf{n}$ be distinct. Then $S_{i j ; k} \approx S_{j i ; k}=\widehat{S}_{i j ; k}$.

Proof By (E1) and (ET4), we have

$$
S_{i j ; k}=X_{k i} X_{i j} X_{j k} \approx \varepsilon_{k} t_{k i} \varepsilon_{i} t_{i j} \varepsilon_{j} t_{j k} \varepsilon_{k} \approx \varepsilon_{k} t_{k j} \varepsilon_{j} t_{j i} \varepsilon_{i} t_{i k} \varepsilon_{k} \approx X_{k j} X_{j i} X_{i k}=S_{j i ; k} .
$$

Lemma 37 Relations (SL1-SL4)' and (RS1-RS4)' are in $\mathscr{Q}^{\sharp}$.

Proof For (SL1) $)^{\prime}$ suppose $1 \leq i<j-1 \leq n-2$. Then

$$
\begin{aligned}
S_{i} L_{j} & =X_{n i} X_{i, i+1} X_{i+1, n} X_{j, j+1} \cdots X_{n-2, n-1} X_{n-1, n} \\
& \approx X_{j, j+1} \cdots X_{n-2, n-1} X_{n i} X_{i, i+1} X_{i+1, n} X_{n-1, n} \\
& \approx X_{j, j+1} \cdots X_{n-2, n-1} X_{n i} X_{i, i+1} \varepsilon_{n-1} X_{i+1, n} \\
& \approx X_{j, j+1} \cdots X_{n-2, n-1} \varepsilon_{n-1} X_{n i} X_{i, i+1} X_{i+1, n} \\
& \approx X_{j, j+1} \cdots X_{n-2, n-1} \varepsilon_{n-1} \varepsilon_{n} X_{n i} X_{i, i+1} X_{i+1, n} \\
& \approx X_{j, j+1} \cdots X_{n-2, n-1} X_{n-1, n} X_{n-1, n} X_{n i} X_{i, i+1} X_{i+1, n} \\
& =L_{j} L_{n-1} S_{i} \\
& \approx L_{n} L_{j} S_{r}
\end{aligned}
$$

by $(\mathrm{L} 1)^{\prime}$.

For $(\mathrm{SL} 2)^{\prime}$ and $(\mathrm{SL} 3)^{\prime}$, we have

$$
\begin{aligned}
S_{i} L_{i+1} & =X_{n i} X_{i, i+1} X_{i+1, n} X_{i+1, i+2} X_{i+2, i+3} \cdots X_{n-1, n} \\
& \approx X_{n i} X_{i, i+1} \varepsilon_{n} X_{i+1, i+2} X_{i+2, i+3} \cdots X_{n-1, n} \\
& \approx X_{n i} \varepsilon_{n} X_{i, i+1} X_{i+1, i+2} X_{i+2, i+3} \cdots X_{n-1, n} \\
& =\varepsilon_{n} t_{n i} \varepsilon_{i} \varepsilon_{n} L_{i} \\
& \approx \varepsilon_{n} t_{n i} \varepsilon_{n} \varepsilon_{i} L_{i} \\
& \approx \varepsilon_{n} L_{i} \\
& =L_{n} L_{i},
\end{aligned}
$$

by Lemma 35

by $(\mathrm{E} 2)$ and $(\mathrm{ET} 1)$

by (ET3) and (E1) 
and

$$
\begin{array}{rlrl}
S_{i} L_{i} & =X_{n i} X_{i, i+1} X_{i+1, n} X_{i, i+1} X_{i+1, i+2} \cdots X_{n-1, n} & \\
& \approx X_{n i} X_{i, i+1} X_{i n} \varepsilon_{i+1} X_{i+1, i+2} \cdots X_{n-1, n} & & \text { by Lemma } 35 \\
& \approx X_{n i} X_{i n} \varepsilon_{i+1} \varepsilon_{i+1} X_{i+1, i+2} \cdots X_{n-1, n} & & \text { by Lemma } 35 \text { again } \\
& \approx \varepsilon_{n} X_{i+1, i+2} \cdots X_{n-1, n} & & \text { by }(25.5) \text { and (E1) } \\
& =L_{n} L_{i+1} . & &
\end{array}
$$$$
\approx X_{n i} X_{i n} \varepsilon_{i+1} \varepsilon_{i+1} X_{i+1, i+2} \cdots X_{n-1, n} \quad \text { by Lemma } 35 \text { again }
$$

For (SL4)', suppose $1 \leq j<i \leq n-2$. We first show that $S_{i} X_{i-1, i} X_{i, i+1} \approx X_{i-1, i} X_{i, i+1} S_{i-1}$. Indeed, we have

$$
\begin{aligned}
S_{i} X_{i-1, i} X_{i, i+1} & =X_{n i} X_{i, i+1} X_{i+1, n} X_{i-1, i} X_{i, i+1} & & \\
& \approx X_{n i} X_{i, i+1} X_{i-1, i} X_{i+1, n} X_{i, i+1} & & \text { by }(25.2) \\
& \approx X_{n i} \varepsilon_{i} X_{i-1, i+1} \varepsilon_{i+1} X_{i n} & & \text { by Lemma } 35 \\
& \approx X_{n i} X_{i-1, i+1} X_{i n} & & \text { by (E1) }
\end{aligned}
$$

while a similar calculation shows that also $X_{i-1, i} X_{i, i+1} S_{i-1} \approx X_{n i} X_{i-1, i+1} X_{i n}$. (This expression simplifies further to $X_{i-1, i+1} \varepsilon_{n}$, although we do not need this.) Now, returning to $(\mathrm{SL} 4)^{\prime}$, we finally calculate

$$
\begin{array}{rlr}
S_{i} L_{j} & =S_{i} X_{j, j+1} \cdots X_{i-2, i-1} X_{i-1, i} X_{i, i+1} L_{i+1} & \\
& \approx X_{j, j+1} \cdots X_{i-2, i-1} S_{i} X_{i-1, i} X_{i, i+1} L_{i+1} & \text { by }(25.2) \\
& \approx X_{j, j+1} \cdots X_{i-2, i-1} X_{i-1, i} X_{i, i+1} S_{i-1} L_{i+1} & \text { by the previous calculation } \\
& \approx X_{j, j+1} \cdots X_{i-2, i-1} X_{i-1, i} X_{i, i+1} L_{n} L_{i+1} S_{i-1} & \text { by (SL1) } \\
& \approx L_{n} X_{j, j+1} \cdots X_{i-2, i-1} X_{i-1, i} X_{i, i+1} L_{i+1} S_{i-1} & \text { by (E2) and (ET1) } \\
& =L_{n} L_{j} S_{i-1} .
\end{array}
$$

Relations (RS1-RS4)' now follow by duality (and Lemma 36).

The next lemma shows how the words $S_{r}$ interact with the generators $t_{i j}$.

Lemma 38 Let $1 \leq r \leq n-2$ and $1 \leq i<j \leq n-1$. Then

$$
S_{r} t_{i j} \approx \begin{cases}t_{i j} S_{r} & \text { if } r \notin\{i-1, i, j-1, j\} \\ t_{i-1, j} S_{r} & \text { if } r=i-1 \\ t_{i+1, j} S_{r} & \text { if } r=i \neq j-1 \\ \varepsilon_{n} t_{i j} & \text { if } r=i=j-1 \\ t_{i, j-1} S_{r} & \text { if } r=j-1 \neq i \\ t_{i, j+1} S_{r} & \text { if } r=j .\end{cases}
$$


Proof Relation (38.1) follows immediately by (29.1). For (38.2), we have

$$
\begin{array}{rlrl}
S_{i-1} t_{i j} & =X_{n, i-1} X_{i-1, i} X_{i n} t_{i j} & \\
& \approx X_{n, i-1} X_{i-1, i} t_{n j} X_{i n} & & \text { by }(29.2) \\
& \approx X_{n, i-1} t_{n j} X_{i-1, i} X_{i n} & & \text { by }(29.1) \\
& \approx t_{i-1, j} X_{n, i-1} X_{i-1, i} X_{i n} & & \text { by }(29.2) \\
& =t_{i-1, j} S_{i-1} . & &
\end{array}
$$

With only one exception, the remaining cases are treated in an almost identical manner. For (38.4), note that for distinct $a, b, c \in \mathbf{n}$, we have

$$
\begin{aligned}
X_{b a} X_{a c} t_{a b} & =\varepsilon_{b} t_{b a} \varepsilon_{a} \varepsilon_{a} t_{a c} \varepsilon_{c} t_{a b} & & \\
& \approx \varepsilon_{b} t_{b a} \varepsilon_{a} t_{a b} t_{a c} \varepsilon_{c} & & \text { by }(\mathrm{E} 1),(\mathrm{T} 2), \text { and }(\mathrm{ET} 1) \\
& \approx \varepsilon_{b} t_{b a} t_{a c} \varepsilon_{c} & & \text { by }(\mathrm{ET} 2) \\
& \approx \varepsilon_{b} t_{b c} t_{a b} \varepsilon_{c} & & \text { by }(\mathrm{T} 2-\mathrm{T} 3) \\
& \approx \varepsilon_{b} t_{b c} \varepsilon_{c} t_{a b} & & \text { by }(\mathrm{ET} 1) \\
& =X_{b c} t_{a b}, & &
\end{aligned}
$$

from which it follows that

$$
\begin{aligned}
S_{a b ; c} t_{a b} & =X_{c a} X_{a b} X_{b c} t_{a b} & & \\
& \approx X_{c a} X_{a b} X_{b a} X_{a c} t_{a b} & & \text { by the observation } \\
& \approx X_{c a} \varepsilon_{a} X_{a c} t_{a b} & & \text { by }(25.5) \\
& \approx X_{c a} X_{a c} t_{a b} & & \text { by }(\mathrm{E} 1) \\
& \approx \varepsilon_{c} t_{a b} & & \text { by }(25.5) .
\end{aligned}
$$

Now (38.4) follows, with $(a, b, c)=(i, i+1, n)$.

Lemma 39 Relations (SA1-SA9)' and $(\mathrm{BS} 1-\mathrm{BS} 9)^{\prime}$ are in $\mathscr{Q}^{\sharp}$.

Proof For $(\mathrm{SA} 1)^{\prime}$ we have

$$
\begin{array}{rlrl}
S_{r} A_{i n} & =X_{n r} X_{r, r+1} X_{r+1, n} t_{i n} \varepsilon_{n} & \\
& \approx X_{n r} X_{r, r+1} X_{r+1, n} \varepsilon_{n} t_{i n} \varepsilon_{n} & & \text { by }(\mathrm{E} 1) \\
& \approx X_{n r} X_{r, r+1} X_{r+1, n} \varepsilon_{n} & & \text { by (ET3) } \\
& \approx X_{n r} X_{r, r+1} X_{r+1, n} & & \text { by (E1) } \\
& =S_{r} . & &
\end{array}
$$


For $(\mathrm{SA} 2)^{\prime}$, if $1 \leq r<i-1<j-1<n-1$, then

$$
\begin{aligned}
S_{r} A_{i j} & =S_{r} t_{i j} L_{j} \\
& \approx t_{i j} S_{r} L_{j} \\
& \approx t_{i j} L_{n} L_{j} S_{r} \\
& \approx L_{n} t_{i j} L_{j} S_{r} \\
& =L_{n} A_{i j} S_{r} .
\end{aligned}
$$

Relations (SA3 - SA9)' are proved analogously, using the relevant part of Lemma 38 and (SL1-SL4)'. Relations (BS1-BS9)' follow by duality.

To tackle relations (S1-S4)' we need some more technical results. The proof of the following lemma also involves our first use of relation (ET5).

Lemma 40 If $i, j, k, l \in \mathbf{n}$ are distinct, then

$$
S_{i j ; k} X_{k l} \approx X_{k l} S_{i j ; l} .
$$

If $i, j, k \in \mathbf{n}$ are distinct, then

$$
S_{i j ; k} X_{k i} \approx X_{k i} S_{k j ; i}
$$

Proof For (40.1), observe first that

$$
X_{k i} X_{i j} X_{j l} X_{l k} \approx \varepsilon_{k} t_{k i} \varepsilon_{i} t_{i j} \varepsilon_{j} t_{j l} \varepsilon_{l} t_{l k} \varepsilon_{k} \approx \varepsilon_{k} t_{k l} \varepsilon_{l} t_{l i} \varepsilon_{i} t_{i j} \varepsilon_{j} t_{j k} \varepsilon_{k} \approx X_{k l} X_{l i} X_{i j} X_{j k}
$$

by (E1) and (ET5). It follows that

$$
\begin{aligned}
S_{i j ; k} X_{k l} & =X_{k i} X_{i j} X_{j k} X_{k l} & & \\
& \approx X_{k i} X_{i j} X_{j k} X_{k l} \varepsilon_{l} & & \text { by }(\mathrm{E} 1) \\
& \approx X_{k i} X_{i j} X_{j k} X_{k l} X_{l j} X_{j l} & & \text { by }(25.5) \\
& \approx X_{k i} X_{i j} X_{j l} X_{l k} X_{k j} X_{j l} & & \text { by Lemma } 36 \\
& \approx X_{k l} X_{l i} X_{i j} X_{j k} X_{k j} X_{j l} & & \text { by the observation } \\
& \approx X_{k l} X_{l i} X_{i j} \varepsilon_{j} X_{j l} & & \text { by (25.5) } \\
& \approx X_{k l} X_{l i} X_{i j} X_{j l} & & \text { by (E1) } \\
& =X_{k l} S_{i j ; l} . & &
\end{aligned}
$$

For (40.2), we have

$$
\begin{aligned}
S_{i j ; k} X_{k i} & \approx S_{j i ; k} X_{k i} & & \text { by Lemma } 36 \\
& =X_{k j} X_{j i} X_{i k} X_{k i} & & \\
& \approx \varepsilon_{k} X_{k j} X_{j i} \varepsilon_{i} & & \text { by (E1) and (25.5) } \\
& \approx X_{k i} X_{i k} X_{k j} X_{j i} & & \text { by (E1) and (25.5) again } \\
& =X_{k i} S_{k j ; i} . & &
\end{aligned}
$$

This completes the proof. 
Lemma 41 Relations $(\mathrm{S} 1-\mathrm{S} 4)^{\prime}$ are in $\mathscr{Q}^{\sharp}$.

Proof Relation (S1) follows immediately from (E1). Next, note that for distinct $a, b, c \in \mathbf{n}$ we have

$$
\begin{aligned}
S_{a b ; c}^{2} & \approx S_{a b ; c} S_{b a ; c} & & \text { by Lemma } 36 \\
& =X_{c a} X_{a b} X_{b c} X_{c b} X_{b a} X_{a c} & & \\
& \approx X_{c a} X_{a b} \varepsilon_{b} X_{b a} X_{a c} & & \text { by }(25.5) \\
& \approx X_{c a} X_{a b} X_{b a} X_{a c} & & \text { by (E1) } \\
& \approx \varepsilon_{c} & & \text { by further reductions using (25.5) and (E1). }
\end{aligned}
$$

Relation $(\mathrm{S} 2)^{\prime}$ follows with $(a, b, c)=(i, i+1, n)$. For $(\mathrm{S} 3)^{\prime}$, fist suppose that $a, b, c, d, e \in \mathbf{n}$ are distinct. Then

$$
\begin{aligned}
S_{a b ; e} S_{c d ; e} & =S_{a b ; e} X_{e c} X_{c d} X_{d e} & & \\
& \approx X_{e c} S_{a b ; c} X_{c d} X_{d e} & & \text { by }(40.1) \\
& \approx X_{e c} X_{c d} S_{a b ; d} X_{d e} & & \text { by }(40.1) \\
& \approx X_{e c} X_{c d} X_{d e} S_{a b ; e} & & \text { by }(40.1) \\
& =S_{c d ; e} S_{a b ; e}, & &
\end{aligned}
$$

and this time $(\mathrm{S} 3)^{\prime}$ follows with $(a, b, c, d, e)=(i, i+1, j, j+1, n)$. For $(\mathrm{S} 4)^{\prime}$, note first that for distinct $a, b, c, d \in \mathbf{n}$, we have

$$
\begin{aligned}
S_{a c ; d} S_{a b ; d} & =S_{a c ; d} X_{d a} X_{a b} X_{b d} & & \\
& \approx X_{d a} S_{d c ; a} X_{a b} X_{b d} & & \text { by }(40.2) \\
& \approx X_{d a} X_{a b} S_{d c ; b} X_{b d} & & \text { by }(40.1) \\
& \approx X_{d a} X_{a b} X_{b d} S_{b c ; d} & & \text { by }(40.2) \\
& =S_{a b ; d} S_{b c ; d} . & &
\end{aligned}
$$

Together with the result of the first calculation above and (E1), this implies that

$$
S_{a b ; d} S_{b c ; d} S_{a b ; d} \approx S_{a c ; d} S_{a b ; d} S_{a b ; d} \approx S_{a c ; d} \varepsilon_{d} \approx S_{a c ; d}
$$

Using this, and Lemma 36, we also have

$$
S_{b c ; d} S_{a b ; d} S_{b c ; d} \approx S_{c b ; d} S_{b a ; d} S_{c b ; d} \approx S_{c a ; d} \approx S_{a c ; d}
$$

These two together imply, with $(a, b, c, d)=(i, i+1, i+2, n)$, that (S4) holds.

The only relations remaining to be covered are $(\mathrm{BL} 1-\mathrm{BL} 6)^{\prime}$ (and also (RA1-RA6)' which will follow by duality). The most difficult to establish is $(\mathrm{RA} 2)^{\prime}$, for which we will need one further technical result. If $r \geq 2$, and $k, i_{1}, \ldots, i_{r} \in \mathbf{n}$ are distinct, we define a word

$$
S_{i_{1}, \ldots, i_{r} ; k}=X_{k i_{1}} X_{i_{1} i_{2}} \cdots X_{i_{r-1} i_{r}} X_{i_{r} k}
$$

noting that this agrees with our earlier definition of $S_{i j ; k}$ when $r=2$. 
Lemma 42 If $r \geq 2$ and $k, i_{1}, \ldots, i_{r} \in \mathbf{n}$ are distinct, then

$$
S_{i_{1}, \ldots, i_{r} ; k} \approx S_{i_{2}, \ldots, i_{r}, i_{1} ; k}
$$

Proof The $r=2$ case is just Lemma 36, and the $r=3$ case was covered in the first calculation of Lemma 40, while if $r \geq 4$, then

$$
\begin{aligned}
& S_{i_{1}, \ldots, i_{r} ; k}=X_{k i_{1}} X_{i_{1} i_{2}} \cdots X_{i_{r-2} i_{r-1}} X_{i_{r-1} i_{r}} X_{i_{r} k} \\
& \approx X_{k i_{1}} X_{i_{1} i_{2}} \cdots X_{i_{r-2} i_{r-1}} X_{i_{r-1} i_{r}} X_{i_{r} k} \varepsilon_{k} \quad \text { by (E1) } \\
& \approx X_{k i_{1}} X_{i_{1} i_{2}} \cdots X_{i_{r-2} i_{r-1}} X_{i_{r-1} i_{r}} X_{i_{r} k} X_{k i_{r-1}} X_{i_{r-1} k} \quad \text { by }(25.5) \\
& \approx X_{k i_{1}} X_{i_{1} i_{2}} \cdots X_{i_{r-2} i_{r-1}} X_{i_{r-1} k} X_{k i_{r}} X_{i_{r} i_{r-1}} X_{i_{r-1} k} \quad \text { by Lemma } 36 \\
& \approx X_{k i_{2}} X_{i_{2} i_{3}} \cdots X_{i_{r-2} i_{r-1}} X_{i_{r-1} i_{1}} X_{i_{1} k} X_{k i_{r}} X_{i_{r} i_{r-1}} X_{i_{r-1} k} \text { by an induction hypothesis } \\
& \approx X_{k i_{2}} X_{i_{2} i_{3}} \cdots X_{i_{r-2} i_{r-1}} X_{i_{r-1} i_{r}} X_{i_{r} i_{1}} X_{i_{1} k} X_{k i_{r-1}} X_{i_{r-1} k} \text { by the } r=3 \text { case } \\
& \approx S_{i_{2}, \ldots, i_{r}, i_{1} ; k} \varepsilon_{k} \quad \text { by }(25.5) \\
& \approx S_{i_{2}, \ldots, i_{r}, i_{1} ; k} \quad \text { by }(\mathrm{E} 1) \text {, }
\end{aligned}
$$

and the proof is complete.

Remark 43 The word $S_{i_{1}, \ldots, i_{r} ; k}$ is mapped to the restriction of the $r$-cycle $\left(i_{1}, \ldots, i_{r}\right)$ to the domain $\mathbf{n} \backslash\{k\}$, so the result of Lemma 42 is natural, given that $\left(i_{2}, \ldots, i_{r}, i_{1}\right)$ represents the same $r$-cycle.

Lemma 44 Let $1 \leq i<j-1 \leq n-1$. Then $B_{i j} L_{i} \approx S_{i} \cdots S_{j-2} \approx S_{i, \ldots, j-1 ; n}$.

Proof We begin by showing that $S_{i} \cdots S_{j-2} \approx S_{i, \ldots, j-1 ; n}$. Now if $j=i+2$, then there is nothing to show, while if $j>i+2$ then, using an induction hypothesis, we have

$$
\begin{aligned}
S_{i} \cdots S_{j-3} S_{j-2} & \approx S_{i, \ldots, j-2 ; n} S_{j-2} \\
& =X_{n i} X_{i, i+1} \cdots X_{j-3, j-2} X_{j-2, n} X_{n, j-2} X_{j-2, j-1} X_{j-1, n} \\
& \approx X_{n i} X_{i, i+1} \cdots X_{j-3, j-2} \varepsilon_{j-2} X_{j-2, j-1} X_{j-1, n} \\
& \approx X_{n i} X_{i, i+1} \cdots X_{j-3, j-2} X_{j-2, j-1} X_{j-1, n} \\
& =S_{i, \ldots, j-1 ; n} .
\end{aligned}
$$

Next, we show that for distinct $k, l, i_{1}, \ldots, i_{r} \in \mathbf{n}$ we have

$$
X_{l k} S_{i_{1}, \ldots, i_{r} ; k} X_{k l} \approx S_{i_{1}, \ldots, i_{r} ; l} .
$$


Indeed, we see first that

$$
\begin{array}{rlrl}
S_{i_{1}, \ldots, i_{r} ; k} X_{k l} & =X_{k i_{1}} X_{i_{1} i_{2}} \cdots X_{i_{r-1} i_{r}} X_{i_{r} k} X_{k l} & \\
& \approx X_{k i_{1}} X_{i_{1} i_{2}} \cdots X_{i_{r-1} i_{r}} X_{i_{r} k} X_{k l} \varepsilon_{l} & & \text { by }(\mathrm{E} 1) \\
& \approx X_{k i_{1}} X_{i_{1} i_{2}} \cdots X_{i_{r-1} i_{r}} X_{i_{r} k} X_{k l} X_{l i_{r}} X_{i_{r} l} & & \text { by }(25.5) \\
& \approx X_{k i_{1}} X_{i_{1} i_{2}} \cdots X_{i_{r-1} i_{r}} X_{i_{r} l} X_{l k} X_{k i_{r}} X_{i_{r} l} & & \text { by Lemma } 36 \\
& \approx X_{k l} X_{l i_{1}} X_{i_{1} i_{2}} \cdots X_{i_{r-1} i_{r}} X_{i_{r} k} X_{k i_{r}} X_{i_{r} l} & & \text { by Lemma } 42 \\
& \approx X_{k l} X_{l i_{1}} X_{i_{1} i_{2}} \cdots X_{i_{r-1} i_{r}} \varepsilon_{i_{r}} X_{i_{r} l} & & \text { by }(25.5) \\
& \approx X_{k l} X_{l i_{1}} X_{i_{1} i_{2}} \cdots X_{i_{r-1} i_{r}} X_{i_{r} l} & & \text { by (E1) } \\
& =X_{k l} S_{i_{1}, \ldots, i_{r} ; l}, & &
\end{array}
$$

and it follows that

$$
\begin{array}{rlrl}
X_{l k} S_{i_{1}, \ldots, i_{r} ; k} X_{k l} & \approx X_{l k} X_{k l} S_{i_{1}, \ldots, i_{r} ; l} & \\
& \approx \varepsilon_{l} S_{i_{1}, \ldots, i_{r} ; l} & & \text { by }(25.5) \\
& \approx S_{i_{1}, \ldots, i_{r} ; l} & & \text { by }(\mathrm{E} 1) .
\end{array}
$$

Finally we calculate that

$$
\begin{array}{rlr}
B_{i j} L_{i} & =R_{j} t_{i j} L_{i} & \\
& =X_{n, n-1} \cdots X_{j+1, j} t_{i j} X_{i, i+1} \cdots X_{j-2, j-1} X_{j-1, j} X_{j, j+1} \cdots X_{n-1, n} & \\
& \approx X_{n, n-1} \cdots X_{j+1, j} \varepsilon_{j} t_{i j} \varepsilon_{i} X_{i, i+1} \cdots X_{j-2, j-1} X_{j-1, j} X_{j, j+1} \cdots X_{n-1, n} & \text { by }(\mathrm{E} 1) \\
& =X_{n, n-1} \cdots X_{j+1, j} X_{j i} X_{i, i+1} \cdots X_{j-2, j-1} X_{j-1, j} X_{j, j+1} \cdots X_{n-1, n} & \\
& =X_{n, n-1} \cdots X_{j+1, j} S_{i, \ldots, j-1 ; j} X_{j, j+1} \cdots X_{n-1, n} & \\
& \approx S_{i, \ldots, j-1 ; n} & \text { by }(44.1)
\end{array}
$$

This last calculation needs to be slightly modified to cover the $j=n$ case; we simply replace the products $X_{n, n-1} \cdots X_{j+1, j}$ and $X_{j, j+1} \cdots X_{n-1, n}$ by $\varepsilon_{j}=\varepsilon_{n}$, and the last line follows by (E1) rather than (44.1). In any case, the proof is now complete.

Lemma 45 Relations (BL1-BL6)' and $(\mathrm{RA} 1-\mathrm{RA} 6)^{\prime}$ are in $\mathscr{Q}^{\sharp}$.

Proof For (BL1)', if $1 \leq k<i<j \leq n$, then by (30.3) and (RL3)' we have

$$
B_{i j} L_{k}=R_{j} t_{i j} L_{k} \approx R_{j} L_{k} t_{i-1, j-1} \approx L_{n} L_{k} R_{j-1} t_{i-1, j-1}=L_{n} L_{k} B_{i-1, j-1} .
$$

Relation (BL2)' was dealt with in the previous lemma. For $(\mathrm{BL} 3)^{\prime}$, if $1 \leq i \leq n-2$, then

$$
\begin{aligned}
B_{i, i+1} L_{i} & =R_{i+1} t_{i, i+1} X_{i, i+1} L_{i+1} & & \\
& \approx R_{i+1} \varepsilon_{i+1} t_{i, i+1} \varepsilon_{i+1} L_{i+1} & & \text { by (E1) and the dual of }(29.3) \\
& \approx R_{i+1} \varepsilon_{i+1} L_{i+1} & & \text { by (ET3) } \\
& \approx R_{i+1} L_{i+1} & & \text { by (E1) } \\
& \approx L_{n} & & \text { by (RL2)'. }
\end{aligned}
$$


If $1 \leq i<k<j \leq n$, then

$$
B_{i j} L_{k}=R_{j} t_{i j} L_{k} \approx R_{j} L_{k} t_{i, j-1} \approx L_{n} L_{k} R_{j-1} t_{i, j-1}=L_{n} L_{k} B_{i, j-1},
$$

so that $(\mathrm{BL} 4)^{\prime}$ holds. For $(\mathrm{BL} 5)^{\prime}$, we have

$$
B_{i j} L_{j}=R_{j} t_{i j} L_{j} \approx R_{j} \varepsilon_{j} t_{i j} \varepsilon_{j} L_{j} \approx R_{j} \varepsilon_{j} L_{j} \approx R_{j} L_{j} \approx L_{n}
$$

Finally, if $1 \leq i<j<k \leq n$, then

$$
B_{i j} L_{k}=R_{j} t_{i j} L_{k} \approx R_{j} L_{k} t_{i j} \approx L_{n} L_{k-1} R_{j} t_{i j}=L_{n} L_{k-1} B_{i j},
$$

showing that (BL6)' holds. Relations (RA1-RA6)' follow by duality.

We have finally dealt with all the relations from $\mathscr{D}^{\prime} \cup \mathscr{R}^{\prime}$, showing that they follow from $\mathscr{Q}$, and have therefore proved the following.

Theorem 46 We have the presentation $\mathcal{P}_{n} \backslash \mathcal{S}_{n} \cong\langle\mathscr{Y} \mid \mathscr{Q}\rangle$.

Remark 47 Theorem 46 has implications for partition algebras. As in [25], we may describe these algebras as twisted semigroup algebras of the partition monoids as follows. Let $F$ denote a commutative ring with identity, and let $\xi \in F$ be an invertible element. Recall that one of the steps in defining the product $\alpha \beta$ of two partitions $\alpha, \beta \in \mathcal{P}_{n}$ involved the removal of any connected components that lived entirely in the middle portion of the concatenated graph. If we denote by $m(\alpha, \beta)$ the number of connected components deleted when forming the product $\alpha \beta$, then the map

$$
\tau: \mathcal{P}_{n} \times \mathcal{P}_{n} \rightarrow F:(\alpha, \beta) \mapsto \xi^{m(\alpha, \beta)}
$$

is a twisting from $\mathcal{P}_{n}$ to $F$. That is, it satisfies the identity

$$
\tau(\alpha, \beta) \tau(\alpha \beta, \gamma)=\tau(\alpha, \beta \gamma) \tau(\beta, \gamma)
$$

This enables us to construct an $F$-algebra $F^{\tau}\left[\mathcal{P}_{n}\right]$, with basis $\mathcal{P}_{n}$, and multiplication $\star$ defined on basis elements by

$$
\alpha \star \beta=\xi^{m(\alpha, \beta)}(\alpha \beta)
$$

and then extended by linearity to combinations of basis elements. This algebra is known as the partition algebra and, without causing confusion, if we allow ourselves to write $\tau$ for the restriction of the twisting to the singular part $\mathcal{P}_{n} \backslash \mathcal{S}_{n}$, the singular part of the partition algebra is $F^{\tau}\left[\mathcal{P}_{n} \backslash \mathcal{S}_{n}\right]$. By [7, Prop. 44], this algebra has (algebra) presentation $\langle\mathscr{Y} \mid \widetilde{\mathscr{Q}}\rangle$ where $\widetilde{\mathscr{Q}}$ is the set of relations obtained from $\mathscr{Q}$ by replacing (E1) by

$$
\varepsilon_{i}^{2}=\xi \varepsilon_{i} \quad \text { for all } i .
$$




\section{References}

[1] J. André. Semigroups that Contain all Singular Transformations. Semigroup Forum, 68(2):304-307, 1966.

[2] G. Ayık, H. Ayık, Y. Ünlü, and J. M. Howie. The Subsemigroup Generated by the Idempotents of a Full Transformation Semigroup. J. London Math. Soc., 41:707-716, 1966.

[3] D. Easdown, J. East, and D. G. FitzGerald. Presentations of Factorizable Inverse Monoids. Acta Sci. Math. (Szeged), 71:509-520, 2005.

[4] D. Easdown, J. East, and D. G. FitzGerald. A Presentation of the Dual Symmetric Inverse Monoid. Internat. J. Algebra Comput., 18(2):357-374, 2008.

[5] J. East. On the Rank of the Singular Part of the Partition Monoids. In preparation.

[6] J. East. A Presentation of the Singular Part of the Symmetric Inverse Monoid. Comm. Alg., 34:1671-1689, 2006.

[7] J. East. Generators and Relations for Partition Monoids and Algebras. Preprint, 2007.

[8] J. East. A Presentation for the Singular Part of the Full Transformation Semigroup. Preprint, 2007.

[9] J. East. Presentations for Singular Subsemigroups of the Partial Transformation Semigroups. Preprint, 2007.

[10] D. G. FitzGerald. A Presentation for the Monoid of Uniform Block Permutations. Bull. Austral. Math. Soc., 68:317-324, 2003.

[11] D. G. FitzGerald and J. Leech. Dual Symmetric Inverse Semigroups and Representation Theory. J. Austral. Math. Soc., 64:146-182, 1998.

[12] G. M. S. Gomes and J. M. Howie. On the Ranks of Certain Finite Semigroups of Transformations. Math. Proc. Cambridge Philos. Soc., 101(3):395-403, 1987.

[13] G. M. S. Gomes and J. M. Howie. On the Ranks of Certain Semigroups of OrderPreserving Transformations. Semigroup Forum, 45(3):272-282, 1992.

[14] T. Halverson and A. Ram. Partition Algebras. European J. Combin., 26(6):869-921, 2005.

[15] P. M. Higgins. Techniques of Semigroup Theory. Oxford Science Publications. The Clarendon Press, Oxford University Press, New York, 1992.

[16] J. M. Howie. The Subsemigroup Generated by the Idempotents of a Full Transformation Semigroup. J. London Math. Soc., 41:707-716, 1966. 
[17] J. M. Howie. An Introduction to Semigroup Theory. L.M.S. Monographs, No. 7. Academic Press, New York, 1976.

[18] J. M. Howie. Idempotent Generators in Finite Full Transformation Semigroups. Proc. Roy. Soc. Edinburgh Sect. A, 81(3-4):317-323, 1978.

[19] J. M. Howie and R. B. McFadden. Idempotent Rank in Finite Full Transformation Semigroups. Proc. Roy. Soc. Edinburgh Sect. A, 114(3-4):161-167, 1990.

[20] K. A. Kearnes, Á. Szendrei, and J. Wood. Generating Singular Transformations. Semigroup Forum, 63(3):441-448, 2001.

[21] M. V. Lawson. Inverse Semigroups. The Theory of Partial Symmetries. World Scientific Publishing Co., Inc., River Edge, NJ, 1998.

[22] S. Lipscombe. Symmetric Inverse Semigroups. American Mathematical Society, Providence, R.I., 1996.

[23] V. Maltcev and V. Mazorchuk. Presentation of the Singular Part of the Brauer Monoid. Math. Bohem., 132(3):297-323, 2007.

[24] M. Petrich. Inverse Semigroups. Pure and Applied Mathematics. Wiley and Sons, 1984.

[25] S. Wilcox. Cellularity of Diagram Algebras as Twisted Semigroup Algebras. J. Algebra, 309(1):10-31, 2007. 